\title{
STELLAR AND PLANETARY PROPERTIES OF K2 CAMPAIGN 1 CANDIDATES AND VALIDATION OF 17 PLANETS, INCLUDING A PLANET RECEIVING EARTH-LIKE INSOLATION
}

\author{
Benjamin T. Montet ${ }^{1,2}$, Timothy D. Morton ${ }^{3}$, Daniel Foreman-Mackey ${ }^{4,5}$, John Asher Johnson ${ }^{2}$, David W. Hogg ${ }^{4,5,6}$, \\ Brendan P. Bowler ${ }^{1,8}$, David W. Latham ${ }^{2}$, Allyson Bieryla ${ }^{2}$, and ANdrew W. ManN ${ }^{7,9}$ \\ ${ }^{1}$ Cahill Center for Astronomy and Astrophysics, California Institute of Technology, Pasadena, CA 91125, USA; btm@astro.caltech.edu \\ ${ }^{2}$ Harvard-Smithsonian Center for Astrophysics, Cambridge, MA 02138, USA \\ ${ }^{3}$ Department of Astrophysics, Princeton University, Princeton, NJ 08544, USA \\ ${ }^{4}$ Center for Cosmology and Particle Physics, Department of Physics, New York University, 4 Washington Place, New York, NY 10003, USA \\ ${ }^{5}$ Center for Data Science, New York University, 726 Broadway, 7th Floor, New York, NY 10003, USA \\ ${ }^{6}$ Max-Planck-Institut für Astronomie, Königstuhl 17, D-69117 Heidelberg, Germany \\ ${ }^{7}$ Department of Astronomy, The University of Texas at Austin, Austin, TX 78712, USA \\ Received 2015 March 27; accepted 2015 July 2; published 2015 August 5
}

\begin{abstract}
The extended Kepler mission, K2, is now providing photometry of new fields every three months in a search for transiting planets. In a recent study, Foreman-Mackey and collaborators presented a list of 36 planet candidates orbiting 31 stars in $K 2$ Campaign 1 . In this contribution, we present stellar and planetary properties for all systems. We combine ground-based seeing-limited survey data and adaptive optics imaging with an automated transit analysis scheme to validate 21 candidates as planets, 17 for the first time, and identify 6 candidates as likely false positives. Of particular interest is K2-18 (EPIC 201912552), a bright $(K=8.9)$ M2.8 dwarf hosting a $2.23 \pm 0.25$ $R_{\oplus}$ planet with $T_{\text {eq }}=272 \pm 15 \mathrm{~K}$ and an orbital period of 33 days. We also present two new open-source software packages which enable this analysis. The first, isochrones, is a flexible tool for fitting theoretical stellar models to observational data to determine stellar properties using a nested sampling scheme to capture the multimodal nature of the posterior distributions of the physical parameters of stars that may plausibly be evolved. The second is vespa, a new general-purpose procedure to calculate false positive probabilities and statistically validate transiting exoplanets.
\end{abstract}

Key words: catalogs - planetary systems - planets and satellites: detection - stars: fundamental parameters

\section{INTRODUCTION}

The Kepler telescope (Borucki et al. 2010) has led to a revolution in stellar and planetary astrophysics, with 7305 "objects of interest" and 4173 "planet candidates" discovered to date (Borucki et al. 2011a, 2011b; Batalha et al. 2013; Burke et al. 2014; Mullally et al. 2015; Rowe et al. 2015). The fidelity of this sample is high: most of these candidates are truly planets (Morton \& Johnson 2011; Fressin et al. 2013; Désert et al. 2015). The mechanical failure of two reaction wheels on the spacecraft led to a repurposing of the spacecraft into the $K 2$ mission, in which the telescope points at fields near the ecliptic plane for $\sim 75$ days at a time (Howell et al. 2014). In this observing strategy, two axes of motion of the spacecraft are controlled by the two remaining reaction wheels, while the roll of the spacecraft is balanced with Solar radiation pressure and quasiperiodic thruster firing. As a result, the detector drifts relative to the sky at the rate of $\sim 1^{\prime \prime} \mathrm{hr}^{-1}$, with rapid corrections due to thruster fires approximately once every six hours. Over the full duration of each campaign, the targets remain near the same location on the detector but both the slow drift and the corrections are observable by eye (Barentsen 2015).

$K 2$ light curves produced with aperture photometry contain substantial pointing-induced photometric variations caused by the star's apparent motion over a poorly defined flat field. Worse yet, these variations occur on timescales similar to transit signals, potentially masking the observational signature of a planet passing between Kepler and its host star.

\footnotetext{
${ }^{8}$ Caltech Joint Center for Planetary Astronomy Fellow.

${ }^{9}$ Harlan J. Smith Fellow.
}

There has been considerable effort to recover these planetary signals, and to date six planets have been confirmed orbiting three stars in the $K 2$ data (Armstrong et al. 2015; Crossfield et al. 2015; Vanderburg et al. 2015). What is common to all of these methods is that removal of systematics is considered a step to be undertaken before the search for planets. Under this strategy, it is implicitly assumed that the systematics are removed perfectly, while retaining all of the astrophysical signal. Of course, it is impossible to perfectly separate the astrophysical and instrumental signal, and such a technique is prone to either over-fitting, in which some of the astrophysical signal is also removed, or under-fitting, in which some of the instrumental systematics remain. A better strategy is to simultaneously fit both the signal and the systematics, as is common practice in cosmology and, increasingly, in radial velocity searches for planetary systems (e.g., Ferreira \& Jaffe 2000; Boisse et al. 2011; Haywood et al. 2014; Grunblatt et al. 2015).

Foreman-Mackey et al. (2015) simultaneously fit both the systematics and potential planetary transit signals in a search for transiting planets. They assume that the dominant trends in the observed stellar light curves are caused by spacecraft motion and are shared by many stars. They then run principal component analysis (PCA) on all stars to measure the dominant modes, modeling each star as a linear combination of 150 of these "eigen light curves" and a transit signal. This method enables fitting without over-fitting, and also permits marginalization over uncertainties induced by the systematic model. Therefore, any uncertainties in the systematics can be propagated into uncertainties in detected planet parameters, instead of assuming the systematics are understood perfectly. 
Using this technique, Foreman-Mackey et al. (2015) detect 36 planet candidates orbiting 31 stars in K2 Campaign 1 data.

In Foreman-Mackey et al. (2015) only transit properties are provided, not absolute parameters about the planet or the star. Additionally, the authors follow the convention of the Kepler team to include any transit event as a candidate system rather than a false positive if a secondary eclipse is not detected: there is no enforced upper limit on the allowed planet radius. The authors intentionally make no effort to separate true transiting planets from astrophysical events that mimic the appearance of transits, such as an eclipsing binary (EB) with a high mass ratio, similar to the Kepler team's list of "objects of interest."

In this paper, we present stellar and planetary parameters for each system. We also analyze the false positive probability (FPP) of each system using vespa, a new publicly available, general-purpose implementation of the Morton (2012) procedure to calculate FPPs for transiting planets. Through this analysis, as well as archival imaging, ground-based seeinglimited survey data, and adaptive optics imaging, we are able to confirm 21 of these systems as transiting planets at the $99 \%$ confidence level. Additionally, we identify six systems as false positives.

This paper is organized as follows. In Section 2, we develop stellar properties through photometric and spectroscopic data. In Section 3, we combine the derived stellar properties with $K 2$ data to infer planet candidate properties. In Section 4, we combine adaptive optics and radial velocity observations with both archival and modern ground-based, seeing limited survey data and an analysis of the transit parameters to calculate FPPs. In Section 5, we discuss potentially interesting systems, including a mini-Neptune orbiting an $\mathrm{M}$ dwarf which receives a similar insolation to the Earth. In Section 6, we summarize and discuss our results.

\section{STELLAR PROPERTIES}

\subsection{Photometry}

With the exception of one star in our sample (K2-18), we do not have spectroscopic data with which to characterize the stellar properties. Additionally, there are no measured parallaxes for any of these stars. Instead, we rely on photometry. For each system, we query the VizieR database of astronomical catalogs (Ochsenbein et al. 2000). We record the $B, V, g^{\prime}, r^{\prime}$, and $i^{\prime}$ magnitudes and their uncertainties from the AAVSO Photometric All-sky Survey DR6 (Henden \& Munari 2014), as reported in the UCAC4 Catalog (Zacharias et al. 2012). We also record the $J, H$, and $K$ magnitudes and their uncertainties as found in the 2MASS All-sky Catalog of Point Sources (Cutri et al. 2003) and the W1-W3 WISE magnitudes and uncertainties from the ALLWise Data Release (Cutri et al. 2013). For all except two of our targets, the $W 4$ band is only an upper limit, and in the remaining two cases, the photometric uncertainity in $W 4$ is at least an order of magnitude larger than those in $W 1-W 3$, so we do not use $W 4$ for any system. These data are reported in Table 1, and a color-color diagram showing the $r-J, J-K$ colors of our candidates is included as Figure 1.

\subsection{Stellar Models}

To convert the observed photometric data into physical properties for each star, we used the new publicly available isochrones Python module, ${ }^{10}$ a general-purpose interpolation tool for the fitting of stellar models to photometric or spectroscopic parameters (Morton 2015a). This software does trilinear interpolation in mass-age- $[\mathrm{Fe} / \mathrm{H}]$ space for any given set of model grids, thus being able to predict the value for any physical or photometric property provided by the models at any values of mass, age, and $[\mathrm{Fe} / \mathrm{H}]$ within the boundaries of the grid.

This enables a set of observed properties $\left(\left\{x_{i}, \sigma_{i}\right\}\right)$, either spectroscopic, photometric, or both, to define a likelihood function to be sampled:

$$
\ln \mathcal{L}(\boldsymbol{\theta}) \propto-\frac{1}{2} \sum_{i} \frac{\left(x_{i}-I_{i}(\boldsymbol{\theta})\right)^{2}}{\sigma_{i}^{2}},
$$

where $I_{i}(\theta)$ is the isochrone model prediction of property $i$ at the given parameters $\boldsymbol{\theta}$. If the observed properties include any apparent magnitudes, then $\boldsymbol{\theta}$ includes distance and extinction in addition to mass, age, and $[\mathrm{Fe} / \mathrm{H}]$.

In this work, we use grids from the Dartmouth Stellar Evolution Database (Dotter et al. 2008) at Solar values of $[\alpha /$ $\mathrm{Fe}]=0.0$ and helium abundance $Y=0.2741$, which come packaged with the isochrones module. We then infer the stellar parameters using MULTINEST (Feroz et al. 2009), an implementation of a multimodal nested sampling algorithm, for each host star conditioned on the observed photometric properties as presented in Table 1. MULTINEST is designed to sample multimodal posteriors, where other samplers such as MCMC algorithms often struggle. Given the multimodal nature of our posteriors, this scheme is optimal for capturing parameter space on the subgiant branch where these stars could reside. We include a prior on stellar metallicity representative of the observed metallicities of stars within $1 \mathrm{kpc}$ of the Sun, following the results of Hayden et al. (2015), and a Salpeter-slope prior on mass up to the maximum mass available in the model grids of $3.7 M_{\odot}$.

During the sampling process, we fit for Galactic extinction as one of our physical parameters. We include the WISE bandpasses by applying the relative extinction values between SDSS, 2MASS, and WISE calculated by Davenport et al. (2014). In each step of our fitting process, we draw a value for $A_{V}$, calculate the expected extinction in all bandpasses $A_{X}$ assuming the $R_{V}=3.1$ reddening law of (Fitzpatrick 1999), and then measure the likelihood of our model stellar fit to the observed apparent magnitudes. We apply a uniform prior ranging from zero to a maximum extinction value of 0.2 and marginalize over extinction in our final determination of stellar parameters. The NASA/IPAC Extragalactic Database, which reports the Schlafly \& Finkbeiner (2011) recalibration of the Schlegel et al. (1998) extinction map as measured by COBE/ DIRBE and IRAS/ISSA, suggests that typical $A_{V}$ extinction values to the edge of the galaxy at this high Galactic latitude are $\sim 0.1 \mathrm{mag}$, so our upper limit appears to be justified.

Such a scheme enables us to infer the statistical uncertainties on the mass, radius, and effective temperature. However, we are subject to biases induced by systematics in the models themselves. There is some evidence that the Dartmouth models may under-predict radii of $\mathrm{M}$ dwarfs by $\sim 15 \%$ when compared to other methods (Montet et al. 2015; Newton et al. 2015). Such an effect may be the result of the Dartmouth model

\footnotetext{
${ }^{10}$ http://github.com/timothydmorton/isochrones
} 
Photometry for All Objects of Interest

\begin{tabular}{|c|c|c|c|c|c|c|c|c|c|c|c|}
\hline EPIC & $B^{\mathrm{a}}$ & $V^{\mathrm{a}}$ & $g^{\mathrm{a}}$ & $r^{\mathrm{a}}$ & $i^{\mathrm{a}}$ & $J^{\mathrm{b}}$ & $H^{\mathrm{b}}$ & $K^{\mathrm{b}}$ & $W 1^{\mathrm{c}}$ & $W 2^{\mathrm{c}}$ & $W 3^{\mathrm{c}}$ \\
\hline 201208431 & $16.23 \pm 0.05$ & $14.91 \pm 0.03$ & $15.56 \pm 0.04$ & $14.29 \pm 0.07$ & $13.89 \pm 0.12$ & $12.37 \pm 0.02$ & $11.75 \pm 0.02$ & $11.57 \pm 0.02$ & $11.51 \pm 0.02$ & $11.55 \pm 0.02$ & $11.58 \pm 0.20$ \\
\hline 201257461 & $12.82 \pm 0.03$ & $11.77 \pm 0.01$ & $12.24 \pm 0.04$ & $11.49 \pm 0.01$ & $11.19 \pm 0.02$ & $9.99 \pm 0.02$ & $9.48 \pm 0.02$ & $9.37 \pm 0.02$ & $9.28 \pm 0.02$ & $9.37 \pm 0.02$ & $9.30 \pm 0.04$ \\
\hline 201295312 & $12.78 \pm 0.04$ & $12.19 \pm 0.12$ & $12.41 \pm 0.03$ & $12.08 \pm 0.09$ & $12.01 \pm 0.21$ & $11.02 \pm 0.03$ & $10.70 \pm 0.02$ & $10.69 \pm 0.02$ & $10.63 \pm 0.02$ & $10.69 \pm 0.02$ & $10.75 \pm 0.12$ \\
\hline 201338508 & $16.30 \pm 0.07$ & $14.91 \pm 0.03$ & $15.62 \pm 0.05$ & $14.33 \pm 0.02$ & $13.79 \pm 0.05$ & $12.45 \pm 0.03$ & $11.76 \pm 0.02$ & $11.60 \pm 0.02$ & $11.49 \pm 0.03$ & $11.49 \pm 0.02$ & $11.16 \pm 0.13$ \\
\hline 201367065 & $13.52 \pm 0.06$ & $12.17 \pm 0.01$ & $12.87 \pm 0.03$ & $11.58 \pm 0.02$ & $10.98 \pm 0.17$ & $9.42 \pm 0.03$ & $8.80 \pm 0.04$ & $8.56 \pm 0.02$ & $8.44 \pm 0.02$ & $8.42 \pm 0.02$ & $8.32 \pm 0.02$ \\
\hline 201384232 & $13.30 \pm 0.05$ & $12.65 \pm 0.04$ & $12.91 \pm 0.05$ & $12.48 \pm 0.06$ & $12.34 \pm 0.07$ & $11.44 \pm 0.02$ & $11.09 \pm 0.02$ & $11.07 \pm 0.02$ & $11.00 \pm 0.02$ & $11.05 \pm 0.02$ & $11.21 \pm 0.16$ \\
\hline 201393098 & $13.90 \pm 0.04$ & $13.21 \pm 0.03$ & $13.54 \pm 0.06$ & $13.02 \pm 0.04$ & $12.85 \pm 0.05$ & $11.95 \pm 0.02$ & $11.63 \pm 0.02$ & $11.56 \pm 0.02$ & $11.52 \pm 0.02$ & $11.57 \pm 0.02$ & $11.61 \pm 0.21$ \\
\hline 201403446 & $12.48 \pm 0.02$ & $12.03 \pm 0.02$ & $12.18 \pm 0.01$ & $11.94 \pm 0.05$ & $11.86 \pm 0.04$ & $11.05 \pm 0.03$ & $10.76 \pm 0.02$ & $10.78 \pm 0.02$ & $10.67 \pm 0.03$ & $10.71 \pm 0.02$ & $10.36 \pm 0.07$ \\
\hline 201445392 & $15.73 \pm 0.02$ & $14.61 \pm 0.03$ & $15.19 \pm 0.04$ & $14.29 \pm 0.02$ & $14.03 \pm 0.07$ & $12.83 \pm 0.03$ & $12.32 \pm 0.03$ & $12.24 \pm 0.03$ & $12.16 \pm 0.02$ & $12.21 \pm 0.02$ & $\ldots$ \\
\hline 201465501 & $\ldots$ & & $16.73 \pm 0.02$ & $15.18 \pm 0.03$ & $14.35 \pm 0.15$ & $12.45 \pm 0.02$ & $11.71 \pm 0.02$ & $11.49 \pm 0.02$ & $11.35 \pm 0.02$ & $11.21 \pm 0.02$ & $11.35 \pm 0.19$ \\
\hline 201505350 & $13.80 \pm 0.02$ & $13.00 \pm 0.01$ & $13.36 \pm 0.02$ & $12.76 \pm 0.01$ & $12.57 \pm 0.02$ & $11.60 \pm 0.02$ & $11.21 \pm 0.02$ & $11.16 \pm 0.03$ & $11.10 \pm 0.02$ & $11.13 \pm 0.02$ & $10.95 \pm 0.12$ \\
\hline 201546283 & $13.51 \pm 0.07$ & $12.64 \pm 0.02$ & $13.03 \pm 0.02$ & $12.37 \pm 0.02$ & $12.17 \pm 0.05$ & $11.16 \pm 0.02$ & $10.79 \pm 0.03$ & $10.70 \pm 0.02$ & $10.61 \pm 0.02$ & $10.66 \pm 0.02$ & $10.53 \pm 0.09$ \\
\hline 201549860 & $15.56 \pm 0.06$ & $14.37 \pm 0.05$ & $14.95 \pm 0.07$ & $13.85 \pm 0.03$ & $13.45 \pm 0.05$ & $12.14 \pm 0.02$ & $11.56 \pm 0.02$ & $11.42 \pm 0.02$ & $11.38 \pm 0.02$ & $11.46 \pm 0.02$ & $11.60 \pm 0.25$ \\
\hline 201555883 & $16.48 \pm 0.01$ & $15.43 \pm 0.01$ & $16.19 \pm 0.10$ & $15.09 \pm 0.13$ & $14.55 \pm 0.08$ & $13.20 \pm 0.02$ & $12.53 \pm 0.03$ & $12.43 \pm 0.03$ & $12.34 \pm 0.02$ & $12.38 \pm 0.03$ & $\ldots$ \\
\hline 201565013 & $\ldots$ & & $18.25 \pm 0.01$ & $16.91 \pm 0.01$ & $16.34 \pm 0.01$ & $14.78 \pm 0.04$ & $14.11 \pm 0.05$ & $14.08 \pm 0.07$ & $13.94 \pm 0.03$ & $13.87 \pm 0.04$ & $\ldots$ \\
\hline 201569483 & $12.90 \pm 0.08$ & $12.05 \pm 0.07$ & $12.44 \pm 0.03$ & $11.76 \pm 0.08$ & $11.48 \pm 0.08$ & $10.39 \pm 0.02$ & $9.97 \pm 0.03$ & $9.88 \pm 0.02$ & $9.82 \pm 0.02$ & $9.87 \pm 0.02$ & $9.82 \pm 0.05$ \\
\hline 201577035 & $13.14 \pm 0.11$ & $12.42 \pm 0.02$ & $12.70 \pm 0.04$ & $12.21 \pm 0.03$ & $12.13 \pm 0.20$ & $11.06 \pm 0.02$ & $10.75 \pm 0.02$ & $10.64 \pm 0.02$ & $10.64 \pm 0.02$ & $10.69 \pm 0.02$ & $10.55 \pm 0.10$ \\
\hline 201596316 & $14.21 \pm 0.01$ & $13.39 \pm 0.09$ & $13.78 \pm 0.07$ & $13.14 \pm 0.12$ & $12.88 \pm 0.10$ & $11.87 \pm 0.02$ & $11.46 \pm 0.02$ & $11.35 \pm 0.02$ & $11.29 \pm 0.02$ & $11.35 \pm 0.02$ & $10.80 \pm 0.11$ \\
\hline 201613023 & $12.99 \pm 0.09$ & $12.26 \pm 0.01$ & $12.56 \pm 0.03$ & $12.05 \pm 0.03$ & $11.96 \pm 0.08$ & $10.98 \pm 0.02$ & $10.71 \pm 0.02$ & $10.61 \pm 0.02$ & $10.58 \pm 0.02$ & $10.63 \pm 0.02$ & $10.59 \pm 0.10$ \\
\hline 201617985 & $16.34 \pm 0.02$ & $14.86 \pm 0.05$ & $15.62 \pm 0.06$ & $14.26 \pm 0.08$ & $13.42 \pm 0.09$ & $11.72 \pm 0.02$ & $11.09 \pm 0.04$ & $10.90 \pm 0.02$ & $10.73 \pm 0.02$ & $10.70 \pm 0.02$ & $10.86 \pm 0.11$ \\
\hline 201629650 & $13.61 \pm 0.03$ & $12.90 \pm 0.04$ & $13.20 \pm 0.03$ & $12.73 \pm 0.01$ & $12.53 \pm 0.06$ & $11.57 \pm 0.03$ & $11.26 \pm 0.02$ & $11.17 \pm 0.03$ & $11.14 \pm 0.02$ & $11.18 \pm 0.02$ & $10.93 \pm 0.12$ \\
\hline 201635569 & $17.74 \pm 0.16$ & $16.31 \pm 0.01$ & $17.02 \pm 0.01$ & $15.62 \pm 0.01$ & $14.87 \pm 0.01$ & $13.42 \pm 0.03$ & $12.77 \pm 0.02$ & $12.61 \pm 0.03$ & $12.52 \pm 0.03$ & $12.55 \pm 0.03$ & $\ldots$ \\
\hline 201649426 & $14.57 \pm 0.03$ & $13.53 \pm 0.01$ & $14.04 \pm 0.01$ & $13.18 \pm 0.02$ & $12.86 \pm 0.06$ & $11.57 \pm 0.02$ & $11.07 \pm 0.02$ & $11.07 \pm 0.02$ & $10.88 \pm 0.02$ & $10.91 \pm 0.02$ & $10.86 \pm 0.12$ \\
\hline 201702477 & $15.27 \pm 0.05$ & $14.57 \pm 0.04$ & $14.89 \pm 0.04$ & $14.40 \pm 0.06$ & $14.24 \pm 0.03$ & $13.27 \pm 0.03$ & $12.88 \pm 0.03$ & $12.77 \pm 0.03$ & $12.81 \pm 0.02$ & $12.84 \pm 0.03$ & $\ldots$ \\
\hline 201736247 & $15.49 \pm 0.06$ & $14.66 \pm 0.05$ & $15.01 \pm 0.04$ & $14.35 \pm 0.04$ & $14.14 \pm 0.02$ & $13.07 \pm 0.02$ & $12.55 \pm 0.02$ & $12.49 \pm 0.03$ & $12.46 \pm 0.02$ & $12.50 \pm 0.02$ & $\ldots$ \\
\hline 201754305 & $15.65 \pm 0.04$ & $14.65 \pm 0.01$ & $15.13 \pm 0.04$ & $14.28 \pm 0.01$ & $13.93 \pm 0.05$ & $12.76 \pm 0.03$ & $12.21 \pm 0.03$ & $12.09 \pm 0.02$ & $12.06 \pm 0.02$ & $12.10 \pm 0.02$ & $12.34 \pm 0.46$ \\
\hline 201779067 & $11.81 \pm 0.01$ & $11.27 \pm 0.01$ & $11.53 \pm 0.07$ & $11.12 \pm 0.01$ & $10.95 \pm 0.01$ & $10.13 \pm 0.02$ & $9.87 \pm 0.02$ & $9.80 \pm 0.02$ & $9.74 \pm 0.02$ & $9.77 \pm 0.02$ & $9.74 \pm 0.04$ \\
\hline 201828749 & $12.48 \pm 0.04$ & $11.76 \pm 0.01$ & $12.13 \pm 0.05$ & $11.58 \pm 0.04$ & $11.32 \pm 0.04$ & $10.49 \pm 0.03$ & $10.23 \pm 0.04$ & $9.93 \pm 0.03$ & $9.82 \pm 0.02$ & $9.87 \pm 0.02$ & $9.98 \pm 0.06$ \\
\hline 201855371 & $14.82 \pm 0.06$ & $13.52 \pm 0.04$ & $14.20 \pm 0.06$ & $12.96 \pm 0.03$ & $12.45 \pm 0.01$ & $11.08 \pm 0.02$ & $10.44 \pm 0.02$ & $10.31 \pm 0.02$ & $10.22 \pm 0.02$ & $10.26 \pm 0.02$ & $10.12 \pm 0.07$ \\
\hline 201912552 & $15.01 \pm 0.06$ & $13.50 \pm 0.05$ & $14.22 \pm 0.05$ & $12.86 \pm 0.04$ & $11.66 \pm 0.08$ & $9.76 \pm 0.03$ & $9.13 \pm 0.03$ & $8.90 \pm 0.02$ & $8.77 \pm 0.02$ & $8.67 \pm 0.02$ & $8.55 \pm 0.03$ \\
\hline 201929294 & $14.32 \pm 0.04$ & $13.31 \pm 0.03$ & $13.78 \pm 0.05$ & $12.97 \pm 0.07$ & $12.61 \pm 0.09$ & $11.48 \pm 0.03$ & $10.98 \pm 0.02$ & $10.80 \pm 0.02$ & $10.73 \pm 0.02$ & $10.78 \pm 0.02$ & $10.67 \pm 0.10$ \\
\hline
\end{tabular}

Notes. These data are available in interactive form at https://filtergraph.com/k2 planets montet.

${ }_{\mathrm{b}}^{\mathrm{a}}$ Magnitude from the AAVSO Photometric All-sky Survey (APASS) DR6 (Henden \& Munari 2014) as reported in the UCAC4 Catalog (Zacharias et al. 2012).

${ }^{\mathrm{b}}$ Magnitude from the 2MASS All-sky Catalog of Point Sources (Cutri et al. 2003).

${ }^{\mathrm{c}}$ Magnitude from the ALLWise Data Release (Cutri et al. 2013). 


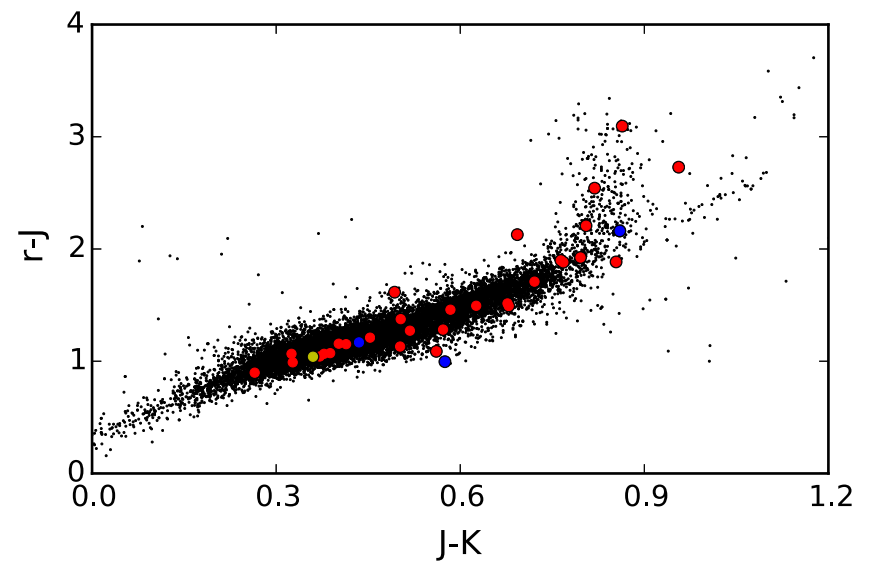

Figure 1. Color-color diagram displaying $r-J, J-K$ photometry for targets observed by Kepler during the original mission (black), with our $K 2$ Campaign 1 planet candidates overlaid (red). Also included is the location of the Sun (yellow) and host stars of previously confirmed $K 2$ planets (blue). $90 \%$ of our candidates have photometry consistent with later spectral types than the Sun.

reliance on BT-Settl atmospheres, which are based on incomplete molecular line lists and have been shown to predict near-IR colors that are too blue (Thompson et al. 2014).

As our stellar results are model-dependent, we caution users who intend to use these parameters for other works, such as exoplanet population studies. When available, stellar parameters inferred through other techniques such as asteroseismology or spectroscopy should supersede these values. We note the observed photometric parameters are consistent with spectroscopically derived parameters for stars with published spectra, and consistent with typical model-dependent uncertainties from photometric data (e.g., Huber et al. 2014). We provide full samples of our posteriors on the physical parameters for each star. ${ }^{11}$

Bastien et al. (2014) use the "granulation flicker" in the Kepler light curves to suggest that approximately $50 \%$ of planet host stars have evolved off the main sequence onto the subgiant branch, so that both the host stars and their planets are larger than previously reported. Similarly, in $K 2$ Campaign 1 we may expect to find evolved stars in a sample of planet candidates, although we may expect the effect to be lessened due to the high Galactic latitude of Campaign 1. Indeed, we find this to be the case. Two stars, EPIC 201257461 and 201649426 are definitively evolved stars, with inferred masses less than $2 M_{\odot}$. but radii above $8 R \cdot$. For approximately one third of the others, we find the stellar radius posterior distribution to be bimodal, with both main sequence and subgiant models of the stars being consistent with the photometric data. This number is consistent with our expectations of the number of subgiant contaminants in the Campaign 1 field (K. Stassun 2015, private communication). Future observations to measure the parallaxes of these stars, such as with Gaia, will be helpful in differentiating between these two models to determine more precisely the stellar, and thus the planetary, radii.

\subsection{SuperNova Integral Field Spectrograph (SNIFS) and SpeX Spectroscopy}

A near-infrared spectrum of K2-18 was obtained using the upgraded SpeX spectrograph (Rayner et al. 2003) on the

\footnotetext{
$11 \mathrm{http}: / / \mathrm{www}$.astro.princeton.edu/ tdm/k2/
}

NASA Infrared Telescope Facility on 2015 January 29 (UT). SpeX observations were taken using the short cross-dispersed mode and the 0 .! $3 \times 15^{\prime \prime}$ slit, which provides simultaneous coverage from 0.7 to $2.5 \mu \mathrm{m}$ at $R \simeq 2000$. The target was observed at two positions along the slit to subsequently subtract the sky background. Eight spectra were taken following this pattern, which provided a final signal-to-noise ratio $(\mathrm{S} / \mathrm{N})$ of $>150$ per resolving element. The spectrum was flat fielded, extracted, wavelength calibrated, and stacked using the Spextool package (Cushing et al. 2004). An A0V-type star was observed immediately after the target, which was used to create a telluric correction using the xtellcor package (Vacca et al. 2003).

An optical spectrum was obtained using SNIFS (Aldering et al. 2002; Lantz et al. 2004) on the University of Hawai'i $2.2 \mathrm{~m}$ telescope on the night of 2015 January 30. SNIFS provides simultaneous coverage from $3200-9700 \AA$ at a resolution of $\simeq 1000$. Final $\mathrm{S} / \mathrm{N}$ of the spectrum was $>100$ per resolving element in the red $(\sim 6000 \AA)$. Details of the SNIFS reduction, including dark, bias, and flat-field corrections, cleaning the data of bad pixels and cosmic rays, and extraction of the one-dimensional spectrum are described in Bacon et al. (2001) and Aldering et al. (2006). Flux calibration was performed using a separate pipeline described in Mann et al. (2015).

$T_{\text {eff }}$ was calculated by comparing our optical spectra with the CFIST suite ${ }^{12}$ of the BT-SETTL version of the PHOENIX atmosphere models (Allard et al. 2013), which gave a temperature of $3503 \pm 60 \mathrm{~K}$. More details of this procedure are given in Mann et al. (2013b) and Gaidos et al. (2014). This method was used because it is known to accurately reproduce empirical $T_{\text {eff }}$ values from long-baseline optical interferometry Boyajian et al. (2012).

Metallcity was determined using the procedures from Mann et al. (2013a), in which the authors provide empirical relations between atomic features and $\mathrm{M}$ dwarf metallicity, calibrated using wide binaries. We adopted the weighted mean of the $H$ and $K$-band calibrations, which yielded a metallicity of 0.09 \pm 0.09 .

We combined the derived $T_{\text {eff }}$ and $[\mathrm{Fe} / \mathrm{H}]$ values with the empirical $T_{\text {eff- }}[\mathrm{Fe} / \mathrm{H}]-R *$ relation from Mann et al. (2015) to compute a radius. Accounting for measurement and calibration errors in $[\mathrm{Fe} / \mathrm{H}]$ and $T_{\text {eff }}$ we calculated a radius $0.394 \pm 0.038 R_{\odot}$. We use these parameters instead of the derived photometric properties for this target, although we note the two are consistent at the $1 \sigma$ level.

The full list of stellar parameters adopted in this paper is included in Table 2.

\section{PLANET PROPERTIES}

In Foreman-Mackey et al. (2015) only parameters directly observable from the $K 2$ light curve itself were reported: the period, time of transit center, and transit depth. With stellar properties now in hand, we can convert these observational results into fundamental parameters of each planet candidate. For each candidate, we fit the light curve using a physical transit model (Mandel \& Agol 2002; Kipping 2010) simultaneously with a systematics model similar to the one described by Foreman-Mackey et al. (2015). We use emcee (ForemanMackey et al. 2013), an implementation of the affine-invariant

\footnotetext{
12 http://phoenix.ens-lyon.fr/Grids/BT-Settl/CIFIST2011/
} 
Table 2

Stellar Properties for All Objects of Interest

\begin{tabular}{|c|c|c|c|c|c|c|c|}
\hline Name & $\begin{array}{c}\text { R.A. (J2000) } \\
\text { (Degrees) }\end{array}$ & $\begin{array}{c}\text { Decl. (J2000) } \\
\text { (Degrees) }\end{array}$ & $\begin{array}{l}\text { Mass } \\
\left(M_{\odot}\right)\end{array}$ & $\begin{array}{c}\text { Radius } \\
\left(R_{\odot}\right)\end{array}$ & $\begin{array}{l}T_{\text {eff }} \\
(\mathrm{K})\end{array}$ & $\begin{array}{c}{[\mathrm{Fe} / \mathrm{H}]} \\
(\mathrm{dex})\end{array}$ & $\begin{array}{c}\text { Distance } \\
(\mathrm{pc})\end{array}$ \\
\hline $201208431 / \mathrm{K} 2-4$ & 174.745639 & -3.905585 & $0.63_{-0.03}^{+0.03}$ & $0.60_{-0.02}^{+0.02}$ & $4197_{-43}^{+45}$ & $-0.12_{-0.12}^{+0.10}$ & $218_{-10}^{+11}$ \\
\hline 201295312 & 174.011629 & -2.520881 & $1.07_{-0.07}^{+0.07}$ & $1.09_{-0.11}^{+0.20}$ & $5989_{-81}^{+100}$ & $-0.02_{-0.18}^{+0.15}$ & $331_{-35}^{+61}$ \\
\hline 201338508/K2-5 & 169.303502 & -1.877976 & $0.53_{-0.01}^{+0.01}$ & $0.52_{-0.01}^{+0.01}$ & $4102_{-41}^{+45}$ & $-0.51_{-0.06}^{+0.04}$ & $181_{-7}^{+7}$ \\
\hline 201367065/K2-3 & 172.334949 & -1.454787 & $0.53_{-0.02}^{+0.02}$ & $0.52_{-0.02}^{+0.02}$ & $3951_{-38}^{+33}$ & $-0.30_{-0.06}^{+0.07}$ & $42_{-2}^{+2}$ \\
\hline 201403446 & 174.266345 & -0.907261 & $1.01_{-0.06}^{+0.08}$ & $1.12_{-0.14}^{+0.26}$ & $6445_{-111}^{+81}$ & $-0.50_{-0.13}^{+0.15}$ & $362_{-48}^{+86}$ \\
\hline 201445392/K2-8 & 169.793666 & -0.284375 & $0.79_{-0.04}^{+0.03}$ & $0.74_{-0.03}^{+0.02}$ & $4890_{-58}^{+38}$ & $-0.01_{-0.13}^{+0.11}$ & $405_{-16}^{+14}$ \\
\hline 201465501/K2-9 & 176.264467 & 0.005301 & $0.24_{-0.03}^{+0.05}$ & $0.25_{-0.03}^{+0.04}$ & $3468_{-19}^{+20}$ & $-0.46_{-0.10}^{+0.12}$ & $66_{-7}^{+11}$ \\
\hline 201505350/K2-19 & 174.960319 & 0.603575 & $0.84_{-0.04}^{+0.04}$ & $0.81_{-0.05}^{+0.09}$ & $5519_{-82}^{+49}$ & $-0.27_{-0.10}^{+0.10}$ & $291_{-20}^{+33}$ \\
\hline 201546283 & 171.515164 & 1.230738 & $0.89_{-0.07}^{+1.15}$ & $0.88_{-0.10}^{+7.37}$ & $5422_{-93}^{+194}$ & $-0.09_{-0.15}^{+0.31}$ & $251_{-29}^{+2138}$ \\
\hline 201577035/K2-10 & 172.121957 & 1.690636 & $0.94_{-0.06}^{+0.04}$ & $0.93_{-0.07}^{+0.16}$ & $5647_{-89}^{+60}$ & $-0.04_{-0.17}^{+0.14}$ & $271_{-21}^{+48}$ \\
\hline 201596316/K2-11 & 169.042002 & 1.986840 & $1.35_{-0.56}^{+0.04}$ & $5.15_{-4.39}^{+0.20}$ & $5433_{-144}^{+49}$ & $-0.12_{-0.17}^{+0.01}$ & $2019_{-1728}^{+71}$ \\
\hline 201613023/K2-12 & 173.192036 & 2.244884 & $1.01_{-0.06}^{+0.05}$ & $1.01_{-0.09}^{+0.27}$ & $5800_{-90}^{+53}$ & $0.03_{-0.17}^{+0.13}$ & $294_{-27}^{+78}$ \\
\hline 201617985 & 179.491659 & 2.321476 & $0.52_{-0.03}^{+0.03}$ & $0.49_{-0.03}^{+0.03}$ & $3742_{-36}^{+31}$ & $-0.08_{-0.11}^{+0.10}$ & $111_{-9}^{+8}$ \\
\hline 201629650/K2-13 & 170.155529 & 2.502696 & $0.80_{-0.04}^{+0.04}$ & $0.78_{-0.05}^{+0.09}$ & $5698_{-82}^{+45}$ & $-0.54_{-0.14}^{+0.12}$ & $290_{-18}^{+34}$ \\
\hline 201635569/K2-14 & 178.057026 & 2.594245 & $0.47_{-0.01}^{+0.01}$ & $0.45_{-0.01}^{+0.01}$ & $3789_{-16}^{+17}$ & $-0.37_{-0.04}^{+0.03}$ & $219_{-8}^{+8}$ \\
\hline 201649426 & 177.234262 & 2.807619 & $1.29_{-0.02}^{+0.02}$ & $8.15_{-0.23}^{+0.32}$ & $5086_{-26}^{+24}$ & $-0.17_{-0.01}^{+0.01}$ & $2537_{-68}^{+92}$ \\
\hline 201702477 & 175.240794 & 3.681584 & $0.87_{-0.06}^{+0.06}$ & $0.85_{-0.08}^{+0.11}$ & $5618_{-85}^{+86}$ & $-0.26_{-0.18}^{+0.17}$ & $673_{-63}^{+87}$ \\
\hline 201736247/K2-15 & 178.110796 & 4.254747 & $0.72_{-0.03}^{+0.06}$ & $0.68_{-0.03}^{+0.06}$ & $5131_{-65}^{+69}$ & $-0.46_{-0.14}^{+0.20}$ & $437_{-22}^{+43}$ \\
\hline 201754305/K2-16 & 175.097258 & 4.557340 & $0.67_{-0.03}^{+0.04}$ & $0.64_{-0.03}^{+0.03}$ & $4761_{-57}^{+50}$ & $-0.40_{-0.17}^{+0.12}$ & $324_{-16}^{+16}$ \\
\hline 201779067 & 168.542699 & 4.988131 & $0.91_{-0.04}^{+0.03}$ & $0.92_{-0.07}^{+0.20}$ & $6166_{-51}^{+30}$ & $-0.54_{-0.12}^{+0.07}$ & $188_{-15}^{+39}$ \\
\hline
\end{tabular}

Notes. These values and their uncertainties are derived from MULTINEST analysis and the numbers are computed as the $0.158,0.500$, and 0.842 posterior sample quantiles. The coordinates are retrieved directly from the EPIC. These data are available in interactive form at https://filtergraph.com/k2_planets_montet.

${ }^{\text {a }}$ Parameters inferred from spectroscopic observations.

ensemble sampler of Goodman \& Weare (2010) to sample from the posterior probability distribution for the stellar-limb darkening coefficients, mass, radius, and effective temperature —and planetary-radius, period, phase, impact parameter, eccentricity, and argument of periapsis-parameters, conditioned on the light curve and the measured stellar properties.

Following Foreman-Mackey et al. (2015) the likelihood function that we use is marginalized over the weights of the "eigen light curves" in the linear systematics model. Unlike Foreman-Mackey et al. (2015) we include an empirical Gaussian prior on the weights determined by robustly computing the distribution of weights across the full set of Campaign 1 light curves. This prior mitigates the incorrect detection of false signals induced by stellar variability-as discussed below in Section 5.2-so we exclude these candidates (EPIC 201929294 and EPIC 201555883) from the tables of results.

In this analysis, we assume the dilution caused by additional stars contributing flux into the aperture is negligible for nearly all systems. Given the location of the Campaign 1 field at a high Galactic latitude, we expect low contamination by background giants. Nevertheless, this assumption may not be valid for all systems. Any contamination unaccounted for, as may happen if any of these stars are actually unresolved binaries, would cause us to underestimate the radii of any planets we detect. Therefore, high-contrast adaptive optics imaging of any systems should be obtained before these planets are used in population inference studies. The planet parameters measured by this analysis are listed in Table 3 .

\section{FALSE POSITIVE ANALYSIS}

There are many scenarios which can cause an astrophysical false positive, where an EB star masquerades as a transiting planet. The most common scenarios are if (a) it is a highly grazing eclipse, or (b) the binary system shares a photometric aperture with a significantly brighter star, resulting in a diluted eclipse depth. When possible, such astrophyscial false positive scenarios are traditionally ruled out by detailed follow-up observations, often a combination of high-resolution imaging and radial-velocity measurements. However, the Kepler mission, with its thousands of planet candidates around 
Table 3

Planet Properties for All Objects of Interest

\begin{tabular}{|c|c|c|c|c|c|c|c|}
\hline Candidate & Period (days) & Epoch (BJD-2456808) & Radius $\left(R_{\oplus}\right)$ & $a / R_{\star}$ & $a(\mathrm{AU})$ & $T_{\text {eq }}(\mathrm{K})$ & Disposition \\
\hline $201208431.01 / \mathrm{K} 2-4 \mathrm{~b}$ & $10.00329 \pm 0.00159$ & $7.5212 \pm 0.0080$ & $2.37 \pm 0.40$ & $27.79 \pm 0.72$ & $0.0777 \pm 0.0012$ & $563 \pm 11$ & Planet \\
\hline 201257461.01 & $50.27762 \pm 0.00785$ & $20.3735 \pm 0.0397$ & $209.52 \pm 99.23$ & $6.19 \pm 0.52$ & $0.3049 \pm 0.0030$ & $1466 \pm 52$ & FP \\
\hline 201295312.01 & $5.65706 \pm 0.00079$ & $3.7187 \pm 0.0082$ & $2.16 \pm 0.57$ & $12.94 \pm 4.07$ & $0.0633 \pm 0.0019$ & $1211 \pm 154$ & Candidate \\
\hline $201338508.01 / \mathrm{K} 2-5 \mathrm{c}$ & $10.93406 \pm 0.00205$ & $6.5947 \pm 0.0080$ & $1.92 \pm 0.20$ & $32.27 \pm 0.71$ & $0.0783 \pm 0.0007$ & $511 \pm 9$ & Planet \\
\hline $201338508.02 / \mathrm{K} 2-5 \mathrm{~b}$ & $5.73491 \pm 0.00061$ & $0.8640 \pm 0.0063$ & $1.92 \pm 0.23$ & $20.99 \pm 0.46$ & $0.0509 \pm 0.0004$ & $634 \pm 12$ & Planet \\
\hline $201367065.01 / \mathrm{K} 2-3 \mathrm{~b}$ & $10.05448 \pm 0.00033$ & $5.4177 \pm 0.0015$ & $1.98 \pm 0.10$ & $30.72 \pm 0.75$ & $0.0740 \pm 0.0009$ & $504 \pm 9$ & Planet \\
\hline $201367065.02 / \mathrm{K} 2-3 \mathrm{c}$ & $24.64745 \pm 0.00152$ & $4.2759 \pm 0.0030$ & $1.56 \pm 0.10$ & $55.85 \pm 1.36$ & $0.1345 \pm 0.0016$ & $374 \pm 7$ & Planet \\
\hline $201384232.01 / \mathrm{K} 2-6 \mathrm{~b}$ & $30.94191 \pm 0.00467$ & $19.5014 \pm 0.0090$ & $2.50 \pm 0.88$ & $50.27 \pm 24.56$ & $0.1898 \pm 0.0056$ & $615 \pm 105$ & Planet \\
\hline 201393098.01/K2-7b & $28.67992 \pm 0.00947$ & $16.6155 \pm 0.0149$ & $2.67 \pm 0.56$ & $40.29 \pm 8.19$ & $0.1814 \pm 0.0043$ & $651 \pm 61$ & Planet \\
\hline 201403446.01 & $19.15344 \pm 0.00607$ & $7.3412 \pm 0.0152$ & $2.04 \pm 0.46$ & $27.05 \pm 5.87$ & $0.1408 \pm 0.0040$ & $889 \pm 88$ & Candidate \\
\hline $201445392.01 / \mathrm{K} 2-8 \mathrm{~b}$ & $10.35176 \pm 0.00133$ & $5.6119 \pm 0.0053$ & $2.97 \pm 0.51$ & $24.94 \pm 0.79$ & $0.0856 \pm 0.0012$ & $691 \pm 14$ & Planet \\
\hline 201445392.02 & $5.06468 \pm 0.00063$ & $5.0663 \pm 0.0071$ & $2.31 \pm 0.33$ & $15.49 \pm 0.49$ & $0.0531 \pm 0.0008$ & $877 \pm 17$ & Candidate \\
\hline $201465501.01 / \mathrm{K} 2-9 \mathrm{~b}$ & $18.44883 \pm 0.00137$ & $14.6723 \pm 0.0030$ & $1.60 \pm 0.42$ & $74.76 \pm 6.66$ & $0.0848 \pm 0.0050$ & $284 \pm 14$ & Planet \\
\hline $201505350.01 / \mathrm{K} 2-19 \mathrm{c}$ & $11.90691 \pm 0.00037$ & $9.2764 \pm 0.0018$ & $4.31 \pm 0.49$ & $24.09 \pm 2.48$ & $0.0965 \pm 0.0017$ & $797 \pm 42$ & Planet \\
\hline $201505350.02 / \mathrm{K} 2-19 \mathrm{~b}$ & $7.91943 \pm 0.00007$ & $5.3836 \pm 0.0005$ & $7.11 \pm 0.81$ & $18.35 \pm 1.89$ & $0.0735 \pm 0.0013$ & $913 \pm 48$ & Planet \\
\hline 201546283.01 & $6.77131 \pm 0.00012$ & $4.8440 \pm 0.0022$ & $5.77 \pm 3.24$ & $17.56 \pm 9.24$ & $0.0668 \pm 0.0029$ & $991 \pm 239$ & Candidate \\
\hline $\begin{array}{l}201549860.01 \\
201555883.01\end{array}$ & $\begin{array}{c}5.60840 \pm 0.00055 \\
\ldots\end{array}$ & $\begin{array}{c}4.1181 \pm 0.0047 \\
\ldots\end{array}$ & $\begin{array}{l}2.20 \pm 0.40 \\
\quad \ldots\end{array}$ & $\begin{array}{c}17.42 \pm 0.46 \\
\ldots\end{array}$ & $\begin{array}{c}0.0555 \pm 0.0008 \\
\ldots\end{array}$ & $\begin{array}{l}766 \pm 14 \\
\quad \ldots\end{array}$ & $\begin{array}{c}\text { Candidate } \\
\mathrm{FP}^{\mathrm{b}}\end{array}$ \\
\hline 201565013.01 & $8.63810 \pm 0.00024$ & $3.4284 \pm 0.0016$ & $15.99 \pm 9.19$ & $28.07 \pm 2.68$ & $0.0669 \pm 0.0031$ & $536 \pm 37$ & Candidate \\
\hline 201569483.01 & $5.79687 \pm 0.00000$ & $5.3135 \pm 0.0004$ & $27.81 \pm 3.56$ & $15.68 \pm 1.91$ & $0.0589 \pm 0.0015$ & $930 \pm 51$ & FP \\
\hline $201577035.01 / \mathrm{K} 2-10 \mathrm{~b}$ & $19.30691 \pm 0.00127$ & $11.5768 \pm 0.0033$ & $3.92 \pm 0.69$ & $32.74 \pm 5.15$ & $0.1374 \pm 0.0025$ & $703 \pm 55$ & Planet \\
\hline $201596316.01 / \mathrm{K} 2-11 \mathrm{~b}$ & $39.93767 \pm 0.23229$ & $21.8290 \pm 0.1156$ & $7.55 \pm 9.33$ & $45.08 \pm 58.53$ & $0.2257 \pm 0.0143$ & $734 \pm 253$ & Planet \\
\hline 201613023.01/K2-12b & $8.28212 \pm 0.00060$ & $7.3734 \pm 0.0054$ & $2.33 \pm 0.58$ & $17.47 \pm 5.05$ & $0.0802 \pm 0.0021$ & $1003 \pm 121$ & Planet \\
\hline 201617985.01 & $7.28161 \pm 0.00078$ & $4.6366 \pm 0.0047$ & $1.78 \pm 0.43$ & $26.04 \pm 1.16$ & $0.0586 \pm 0.0012$ & $518 \pm 16$ & Candidate \\
\hline $201629650.01 / \mathrm{K} 2-13 \mathrm{~b}$ & $39.91488 \pm 0.32477$ & $4.5250 \pm 0.0146$ & $1.89 \pm 0.95$ & $79.69 \pm 63.37$ & $0.2114 \pm 0.0061$ & $511 \pm 126$ & Planet \\
\hline $201635569.01 / \mathrm{K} 2-14 \mathrm{~b}$ & $8.36802 \pm 0.00019$ & $3.4513 \pm 0.0013$ & $4.81 \pm 0.42$ & $30.16 \pm 0.69$ & $0.0627 \pm 0.0006$ & $488 \pm 8$ & Planet \\
\hline 201649426.01 & $27.77045 \pm 0.00008$ & $13.3482 \pm 0.0012$ & $32.79 \pm 9.01$ & $59.26 \pm 13.58$ & $0.1517 \pm 0.0097$ & $441 \pm 42$ & FP \\
\hline 201702477.01 & $40.73620 \pm 0.00266$ & $3.5455 \pm 0.0025$ & $7.28 \pm 1.10$ & $56.98 \pm 7.61$ & $0.2205 \pm 0.0053$ & $529 \pm 36$ & Candidate \\
\hline 201736247.01/K2-15b & $11.81040 \pm 0.00204$ & $3.8509 \pm 0.0076$ & $2.48 \pm 0.30$ & $28.84 \pm 1.98$ & $0.0910 \pm 0.0018$ & $676 \pm 26$ & Planet \\
\hline 201754305.01/K2-16c & $19.07536 \pm 0.00490$ & $1.4854 \pm 0.0119$ & $2.14 \pm 0.41$ & $41.43 \pm 1.34$ & $0.1220 \pm 0.0021$ & $523 \pm 12$ & Planet \\
\hline $201754305.02 / \mathrm{K} 2-16 \mathrm{~b}$ & $7.62067 \pm 0.00095$ & $3.6802 \pm 0.0054$ & $2.13 \pm 0.37$ & $22.47 \pm 0.73$ & $0.0662 \pm 0.0011$ & $710 \pm 16$ & Planet \\
\hline 201779067.01 & $27.24273 \pm 0.00012$ & $12.2601 \pm 0.0003$ & $31.73 \pm 5.25$ & $38.25 \pm 3.72$ & $0.1718 \pm 0.0022$ & $707 \pm 34$ & FP \\
\hline 201828749.01 & $33.51569 \pm 0.00232$ & $5.1504 \pm 0.0034$ & $3.83 \pm 3.25$ & $67.09 \pm 67.64$ & $0.1875 \pm 0.0090$ & $613 \pm 239$ & Candidate \\
\hline 201855371.01/K2-17b & $17.96753 \pm 0.00152$ & $9.9462 \pm 0.0035$ & $2.23 \pm 0.20$ & $39.38 \pm 0.85$ & $0.1190 \pm 0.0020$ & $487 \pm 10$ & Planet \\
\hline $\begin{array}{l}201912552.01 / \mathrm{K} 2-18 \mathrm{~b}^{\mathrm{a}} \\
201929294.01\end{array}$ & $32.94488 \pm 0.00281$ & $\begin{array}{c}28.1849 \pm 0.0027 \\
\ldots\end{array}$ & $2.24 \pm 0.23$ & $\begin{array}{c}83.83 \pm 9.03 \\
\ldots\end{array}$ & $\begin{array}{c}0.1491 \pm 0.0055 \\
\ldots\end{array}$ & $272 \pm 15$ & $\begin{array}{c}\text { Planet } \\
\text { FP }^{\mathrm{b}}\end{array}$ \\
\hline
\end{tabular}

Notes. These values and uncertainties are given by the mean and standard deviation of MCMC posterior samplings. These data are available in interactive form at https://filtergraph.com/k2_planets_montet.

${ }^{\text {a }}$ Parameters inferred from spectroscopic observations.

${ }^{\mathrm{b}}$ Declared a false positive due to noise modeling systematics (see Section 5.2).

mostly faint stars, necessitated a paradigm shift-a move toward probabilistic interpretation of transit signals, rather than comprehensive follow-up of each individual candidate (Morton \& Johnson 2011).

Morton (2012) presented an automated method to calculate the probability that a planet candidate might be caused by an astrophysical false positive. This method uses Galactic population simulations to determine the distributions of possible false positive scenarios, comparing the typical light curve shape of each to the data. It then combines this information with observationally motivated prior assumptions about the populations of field stars, the properties of multiple star systems, and the occurrence rate of planets as determined from Kepler (Fressin et al. 2013), in order to determine the probability that the observed signal may be a false positive. Similar in spirit to other published methods of probabilistic validation, such as BLENDER (Torres et al. 2011) and PASTIS (Díaz et al. 2014), it has the advantage of being computationally less demanding and fully automated, and thus easily applied in batch to a large number of candidates.
In this work, we use vespa ${ }^{13}$ (Morton 2015b), a new publicly available, general-purpose implementation of the Morton (2012) procedure, to calculate FPPs for each of these $K 2$ candidates. The following constraints on false positive scenarios are imposed.

1. A chance-aligned EB system may reside anywhere inside or within one pixel of the photometric aperture of the target star. In creating a light curve for each star, we define photometric apertures ranging from 10 to 20 arcsec for each star, as defined in Table 4. Given the 6 arcsec point-spread function (PSF) of the Kepler telescope, we allow for the possibility that companions falling just outside of our aperture (within one pixel) may contribute to the light curve, possibly causing a false positive event. The search for such companions is discussed in Section 4.3.

\footnotetext{
${ }^{13}$ http://github.com/timothydmorton/vespa
} 
Table 4

Detected Companions to Candidate Host Stars

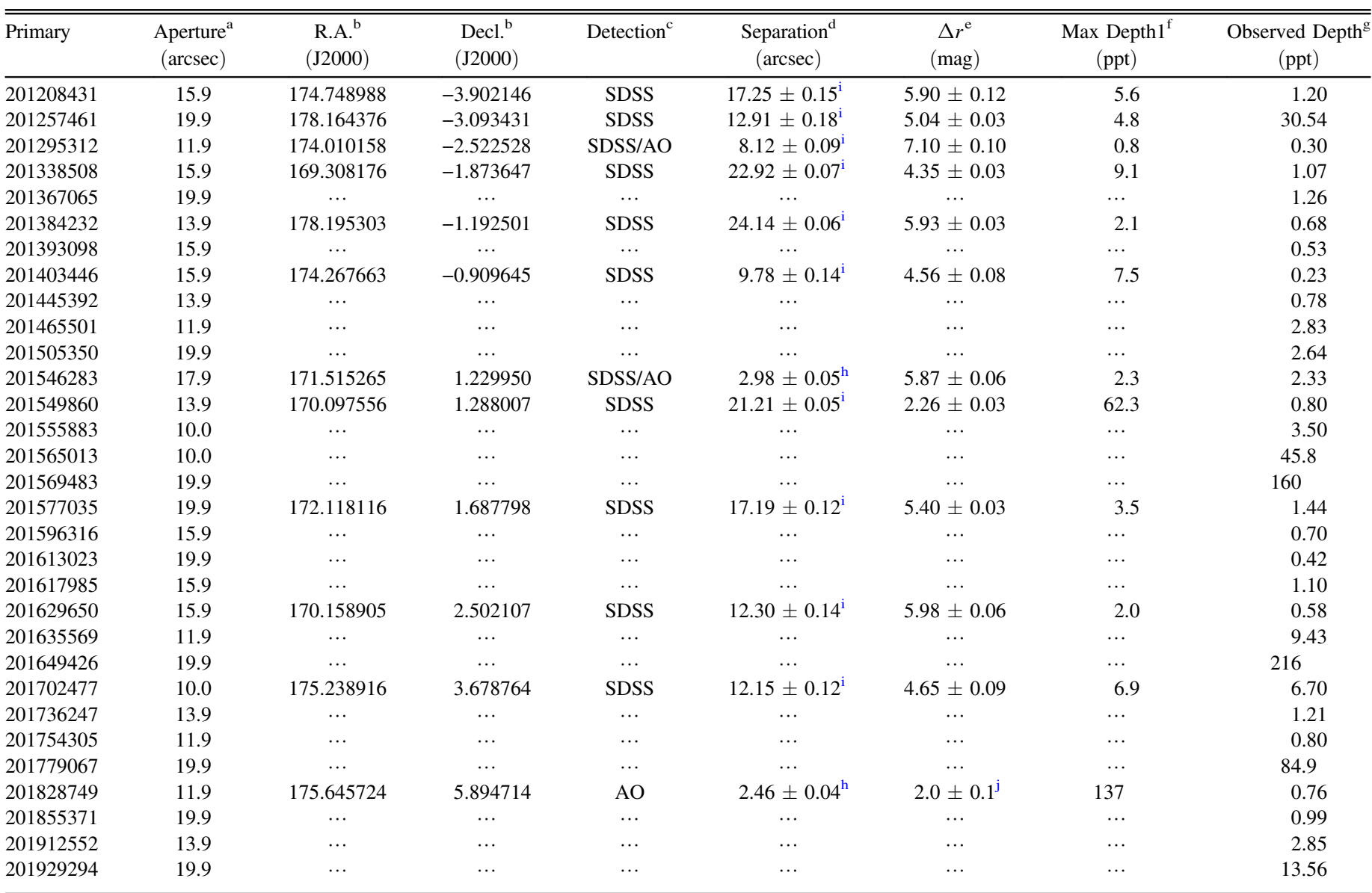

Notes.

a Defined aperture used to create the $K 2$ stellar light curve.

b Position of imaged companion.

${ }^{\mathrm{c}}$ Dataset used to detect the imaged companion.

d Distance between the primary $K 2$ target star and companion, in the dataset in which the companion is detected.

e Difference in $r$-band magnitude between the primary $K 2$ target star and the companion.

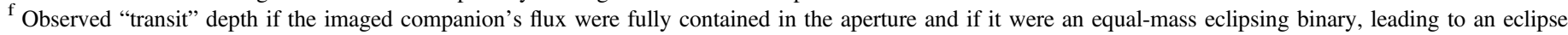
depth of $50 \%$. This is the maximum possible false positive eclipse depth, as described in Section 4.3.

g Observed transit depth in the $K 2$ dataset. If larger than the "max depth," this transit event cannot be caused by eclipses of the background star.

h Separation from AO imaging.

${ }^{\mathrm{i}}$ Separation from SDSS photometry.

${ }^{\mathrm{j}} \Delta r$ inferred from $J H K$ relative photometry.

2. The maximum allowed depth of a potential secondary eclipse event is the most significantly detected signal at the same period of the planet candidate, once the primary transit is masked out (discussed in Section 4.1). vespa does not allow for the possibility of secondary eclipses larger than those observed in the $K 2$ light curve for each star.

3. Blended stars must be allowed by the available adaptive optics and archival imaging data (discussed in detail in Sections 4.2 and 4.3 ). vespa only considers stars below the detection threshold for the AO imaging, which is a position-dependent value following a calculated contrast curve for each star.

Each of these scenarios is an astrophysical eclipse, caused by one object passing in front of another, blocking some fraction of the total light. The calculations here do not include the possibility that each signal is caused by an instrumental artifact in the data or some other astrophysical event, such as stellar activity, masquerading as planet transits.

Table 5 summarizes the results of these calculations, presenting the relative probability for each candidate to be caused by any of three false positive scenarios: an undiluted EB, a hierarchical triple eclipsing binary (HEB), and a chancealigned background(/foreground) eclipsing binary (BEB).

Six of the presented candidates have FPP $>90 \%$; these are considered to be likely false positives. On the other hand, 24 candidates have FPP $<1 \%$. Three of the transit signals might plausibly be caused by contamination by detected stellar companions within the photometric apertures (see Section 4.3), so we keep these as candidates.

This leaves 21 candidates that we statistically validate as planets, including four that have been previously identified in the literature (Armstrong et al. 2015; Crossfield et al. 2015). So 
Table 5

False Positive Probability Calculation Results

\begin{tabular}{|c|c|c|c|c|c|c|c|c|}
\hline Candidate & $\delta_{\text {sec, } \max }(\mathrm{ppt})^{\mathrm{a}}$ & $\mathrm{AO} ?^{\mathrm{b}}$ & $\operatorname{Pr}_{\mathrm{EB}}$ & $\operatorname{Pr}_{\mathrm{BEB}}$ & $\operatorname{Pr}_{\mathrm{HEB}}$ & $f_{p}^{\mathrm{c}}$ & FPP & Disposition \\
\hline 201257461.01 & 0.59 & $\cdots$ & 0.998 & $1.7 \times 10^{-3}$ & $<10^{-4}$ & 0.00 & 1.000 & FP \\
\hline 201295312.01 & 0.04 & $\mathrm{Y}$ & $1.4 \times 10^{-4}$ & $<10^{-4}$ & $<10^{-4}$ & 0.17 & $1.4 \times 10^{-4}$ & Candidate $^{\mathrm{d}}$ \\
\hline 201338508.02/K2-5b & 0.33 & $\ldots$ & $<10^{-4}$ & $1.7 \times 10^{-4}$ & $<10^{-4}$ & 0.22 & $1.7 \times 10^{-4}$ & Planet \\
\hline $201367065.01 / \mathrm{K} 2-3 \mathrm{~b}$ & 0.15 & $\cdots$ & $<10^{-4}$ & $1.1 \times 10^{-4}$ & $<10^{-4}$ & 0.22 & $1.1 \times 10^{-4}$ & Planet $^{\mathrm{f}}$ \\
\hline $201367065.02 / \mathrm{K} 2-3 \mathrm{c}$ & 0.67 & $\cdots$ & $<10^{-4}$ & $<10^{-4}$ & $<10^{-4}$ & 0.16 & $<10^{-4}$ & Planet $^{\mathrm{f}}$ \\
\hline 201403446.01 & 0.18 & $\mathrm{Y}$ & $4.8 \times 10^{-4}$ & $<10^{-4}$ & $<10^{-4}$ & 0.19 & $4.9 \times 10^{-4}$ & Candidate $^{\mathrm{d}}$ \\
\hline 201445392.01/K2-8b & 0.26 & $\ldots$ & $<10^{-4}$ & $2.1 \times 10^{-3}$ & $<10^{-4}$ & 0.18 & $2.1 \times 10^{-3}$ & Planet \\
\hline 201445392.02 & 0.18 & $\ldots$ & $<10^{-4}$ & 0.019 & $<10^{-4}$ & 0.21 & 0.019 & Candidate \\
\hline 201465501.01/K2-9b & 0.68 & $\cdots$ & $<10^{-4}$ & $5.8 \times 10^{-3}$ & $<10^{-4}$ & 0.21 & $5.8 \times 10^{-3}$ & Planet \\
\hline $201505350.01 / \mathrm{K} 2-19 \mathrm{c}$ & 2.69 & $\cdots$ & $<10^{-4}$ & $5.6 \times 10^{-3}$ & $<10^{-4}$ & 0.04 & $5.6 \times 10^{-3}$ & Planet $^{g}$ \\
\hline $201505350.02 / \mathrm{K} 2-19 \mathrm{~b}$ & 0.70 & $\cdots$ & $<10^{-4}$ & $1.6 \times 10^{-4}$ & $<10^{-4}$ & 0.07 & $1.7 \times 10^{-4}$ & Planet $^{\mathrm{g}}$ \\
\hline 201569483.01 & 2.06 & $\cdots$ & 0.822 & $<10^{-4}$ & 0.174 & 0.00 & 0.996 & FP \\
\hline $201577035.01 / \mathrm{K} 2-10 \mathrm{~b}$ & 0.14 & Y & $4.4 \times 10^{-4}$ & $<10^{-4}$ & $<10^{-4}$ & 0.07 & $4.4 \times 10^{-4}$ & Planet \\
\hline $201596316.01 / \mathrm{K} 2-11 \mathrm{~b}$ & 0.45 & $\ldots$ & $<10^{-4}$ & $1.2 \times 10^{-3}$ & $<10^{-4}$ & 0.06 & $1.2 \times 10^{-3}$ & Planet \\
\hline $201613023.01 / \mathrm{K} 2-12 \mathrm{~b}$ & 0.08 & $\mathrm{Y}$ & $<10^{-4}$ & $<10^{-4}$ & $<10^{-4}$ & 0.18 & $<10^{-4}$ & Planet \\
\hline 201617985.01 & 0.27 & $\ldots$ & $<10^{-4}$ & 0.012 & $<10^{-4}$ & 0.18 & 0.012 & Candidate \\
\hline $201629650.01 / \mathrm{K} 2-13 \mathrm{~b}$ & 0.43 & $\ldots$ & $5.9 \times 10^{-4}$ & $2.0 \times 10^{-4}$ & $<10^{-4}$ & 0.13 & $7.8 \times 10^{-4}$ & Planet \\
\hline $201635569.01 / \mathrm{K} 2-14 \mathrm{~b}$ & 0.79 & $\cdots$ & $<10^{-4}$ & $4.9 \times 10^{-3}$ & $<10^{-4}$ & 0.05 & $4.9 \times 10^{-3}$ & Planet \\
\hline 201649426.01 & 3.10 & $\cdots$ & 0.896 & $<10^{-4}$ & 0.104 & 0.00 & 1.000 & FP \\
\hline 201702477.01 & 0.70 & $\cdots$ & 0.137 & $1.2 \times 10^{-3}$ & $6.6 \times 10^{-3}$ & 0.05 & 0.145 & Candidate \\
\hline $201736247.01 / \mathrm{K} 2-15 \mathrm{~b}$ & 0.42 & $\cdots$ & $4.8 \times 10^{-4}$ & $2.1 \times 10^{-4}$ & $<10^{-4}$ & 0.19 & $6.9 \times 10^{-4}$ & Planet \\
\hline $201754305.01 / \mathrm{K} 2-16 \mathrm{c}$ & 0.65 & $\cdots$ & $1.0 \times 10^{-4}$ & $1.4 \times 10^{-3}$ & $<10^{-4}$ & 0.21 & $1.5 \times 10^{-3}$ & Planet \\
\hline 201754305.02/K2-16b & 0.38 & $\cdots$ & $2.3 \times 10^{-4}$ & $9.9 \times 10^{-4}$ & $<10^{-4}$ & 0.19 & $1.2 \times 10^{-3}$ & Planet \\
\hline 201779067.01 & 1.97 & $\cdots$ & 0.968 & $1.3 \times 10^{-3}$ & $7.2 \times 10^{-3}$ & 0.00 & 0.976 & FP \\
\hline
\end{tabular}

Notes. Results of the vespa astrophysical false positive probability calculations for all candidates. Likely false positives (FPP $>0.9$, or otherwise designated) are marked in red. Candidates are declared to be validated planets if FPP $<0.01$. EB, BEB, and HEB refer to the three considered astrophysical false positive scenarios, and the relative probability of each is listed in the appropriate column. Planets previously identified in the literature are marked.

${ }^{\text {a }}$ Maximum depth of potential secondary eclipse signal.

${ }^{\mathrm{b}}$ Whether adaptive optics observation is presented in this paper.

${ }^{c}$ Integrated planet occurrence rate assumed between $0.7 \times$ and $1.3 \times$ the candidate radius

${ }^{\mathrm{d}}$ Despite low FPP, returned to candidate status out of abundance of caution due to secondary star detection within or near photometric aperture.

${ }^{\mathrm{e}}$ Declared a false positive due to noise modeling systematics (see Section 5.2).

${ }^{\mathrm{f}}$ Identified as planets by Crossfield et al. (2015).

g Identified as planets by Armstrong et al. (2015).

in total, of the 36 candidates, 21 are secure planets, 17 of which we validate here for the first time.

We emphasize that the majority of these validations rely solely on the transit photometry and SDSS data, with follow-up imaging only obtained for seven of the 31 targets. This demonstrates the utility of the vespa tool, which will be crucial to interpreting future candidates detected by $K 2, T E S S$, and PLATO and prioritizing follow-up observing efforts. We show the transit signals in Figure 2.

\subsection{Secondary Eclipse Observations}

One of the definitive signatures of a false positive binary star system masquerading as a transiting planet is the presence of a secondary eclipse. While a nondetection of a secondary does not exclude the possibility of a binary system (the orbit may be eccentric, or the companion too faint for a secondary eclipse to be detectable in the noise), such a nondectection reduces the probability of each of the EB false positive scenarios.

To attempt to eliminate each EB scenario, we first search each $K 2$ light curve to determine which secondary eclipse signals are not allowed by the data. We mask the transit signal of the planet in question and search for the most significant signal at the same period. Such a scheme does not assume circular orbits: we return the most significant signal at any phase, not only at the midpoint between consecutive transits.

We report these maximum allowable secondary eclipse depths in Table 5. These values are used by vespa as limits on the allowable secondary eclipse. Any models that cause a larger event, such as a background EB consisting of two equal-mass 

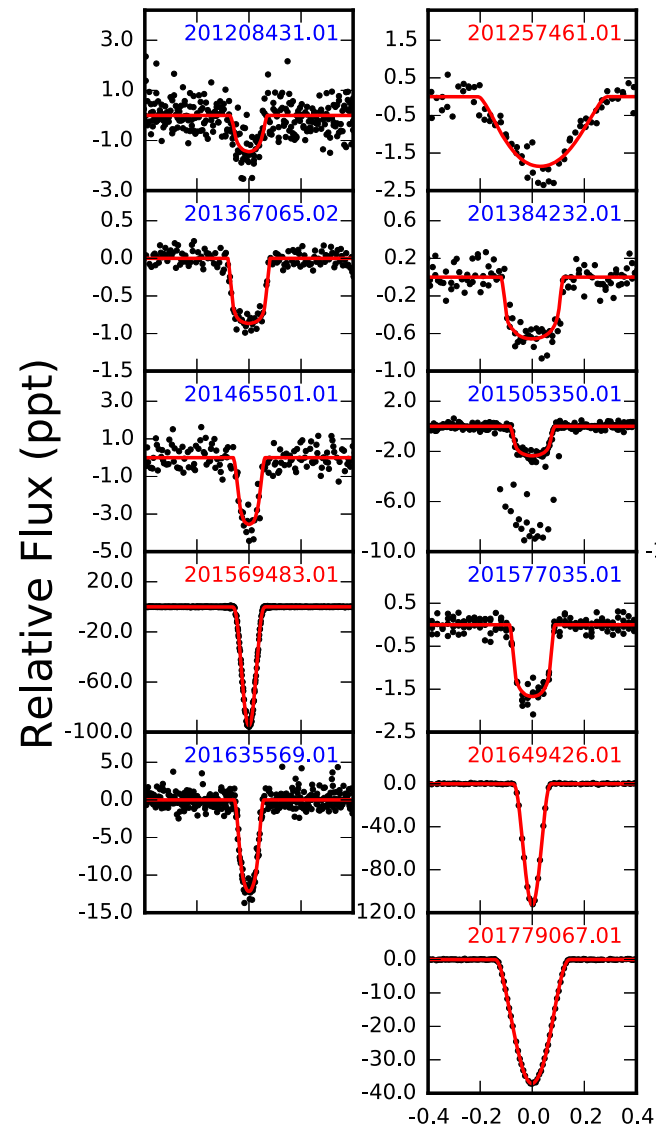
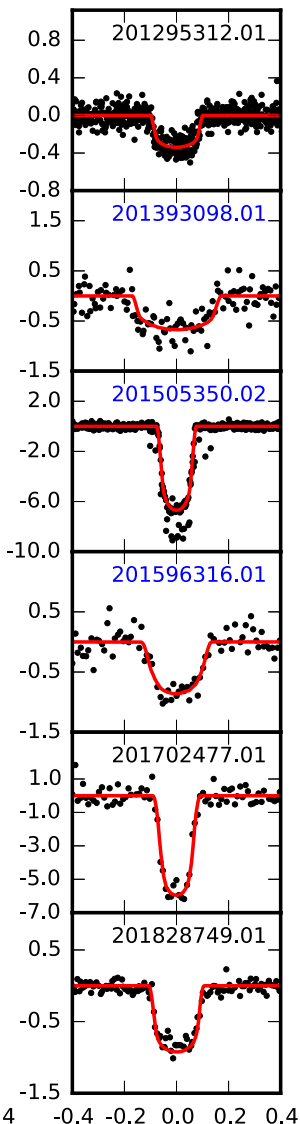

Time from Transit Center (Days)
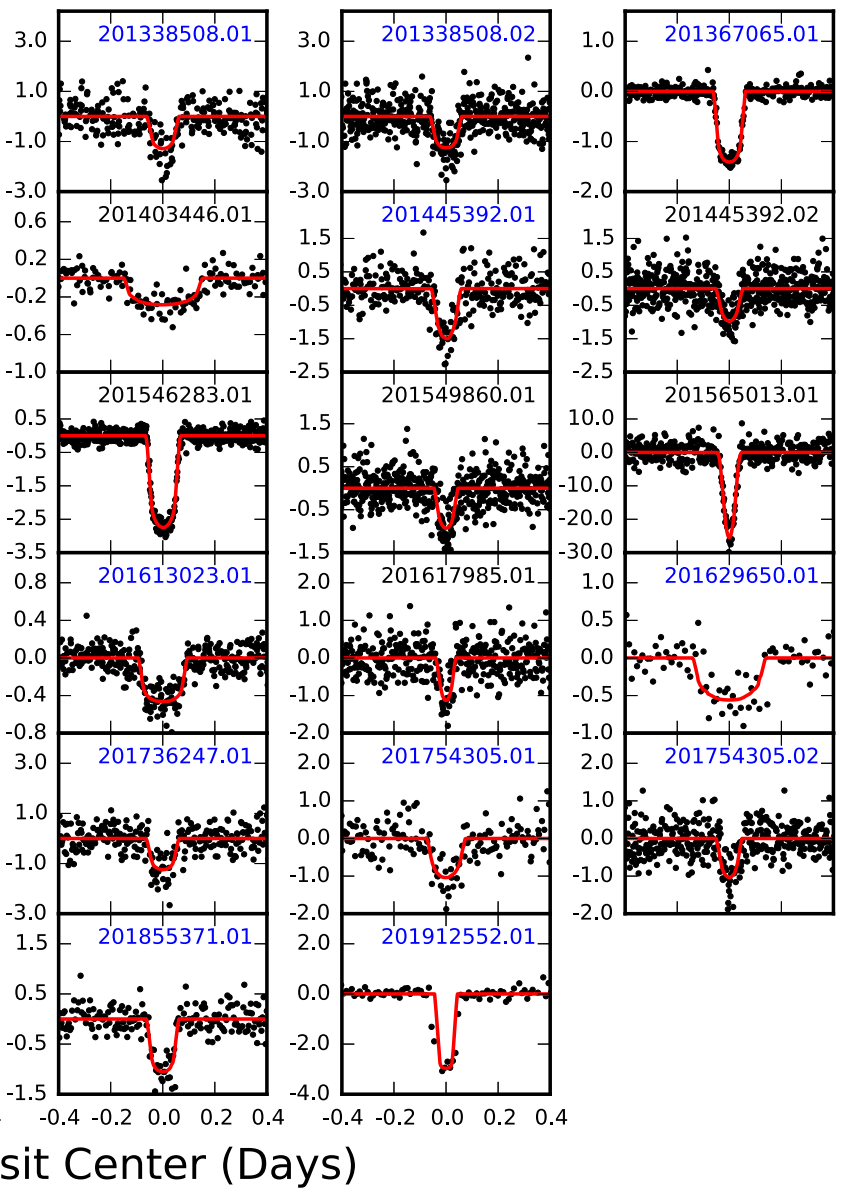

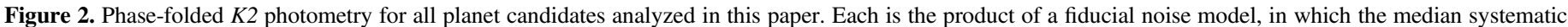

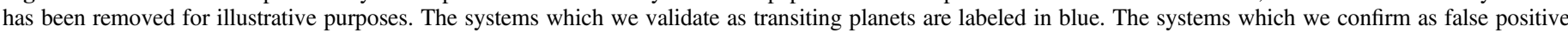

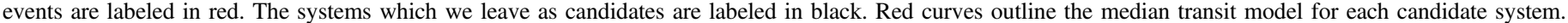

stars in a circular orbit, can be excluded by the data. We note that with the exception of K2-19c (EPIC 201505350.01), all systems with a maximum eclipse depth of at least one part per thousand have FPPs of 0.866 or larger. The exception, K2-19, is a two-planet system with the two planets near a 3:2 period commensurability, so in this case the "secondary" is actually the transits of the other planet.

\subsection{Adaptive Optics Imaging}

We obtained high resolution images of seven stars with the Palomar High Angular Resolution Observer (PHARO) infrared detector (Hayward et al. 2001) behind the PALM3000 adaptive optics system (Dekany et al. 2013) at the Palomar 5.1 m Hale telescope on the nights of 2015 February 3 and 4 UT. Sky conditions were mostly clear with light cirrus and $\approx 1$ ". $0-1$ ". 3 seeing on both nights. We used the smallest plate scale of 25 mas pixels $^{-1}$ which resulted in a field of view of $25^{\prime \prime} .6 \times 25^{\prime \prime} .6$ across the $1024^{2}$ pixels ${ }^{2}$ array. All observations were obtained with the $32 \mathrm{x}$ pupil sampling mode, resulting in Strehl ratios of $\approx 20 \%-30 \%$ in $K_{S}$ for our $V=11-13 \mathrm{mag}$ targets as measured by the Strehl monitor at the telescope in real time. We obtained unsaturated dithered frames of each target in $K_{S}$-band with typical integration times of 2-10 s. Except for EPIC 201828749 and EPIC 201546283, which had nearby candidate binary companions, we also acquired deep saturated images (5-10 frames at $60 \mathrm{~s}$ each) to search for fainter companions.

Images were registered and contrast curves were generated following Bowler et al. (2015). For the saturated data, the star's position in each image was found by masking the saturated region and fitting a 2D bivariate Gaussian to the PSF wings. Contrast curves for the median-combined image are calibrated using the unsaturated frames. The typical sensitivity is 6.5-7.5 mag at $1^{\prime \prime}$. The images were astrometrically calibrated using dithered observations of the Trapezium cluster centered on $\theta^{1}$ Ori $\mathrm{C}$ taken on 2015 February 3 UT. Based on the reference astrometry for pairs of stars in the field from McCaughrean \& Stauffer (1994), we measure a plate scale of $25.2 \pm 0.4$ mas pixels $^{-1}$ and north orientation of $-0.2 \pm 0.3$. Since this latter value is consistent with being aligned with the detector columns, we adopt a value of $0: 0 \pm 0: 3$ for this work. Relative photometry of nearby stars is carried out using aperture photometry with an aperture radius of 12 pixels $(0$." 3$)$. For EPIC 201828749, we also acquired $J$ - and $H$-band images. Astrometry and photometry is derived separately for each image, and the mean and standard deviation of these measurements is adopted for our final values listed in Table 4.

Images for all systems AO data was obtained for is shown in Figure 3, while contrast curves showing the $5 \sigma$ limits for detection as a function of orbital separation are given in Figure 4. 


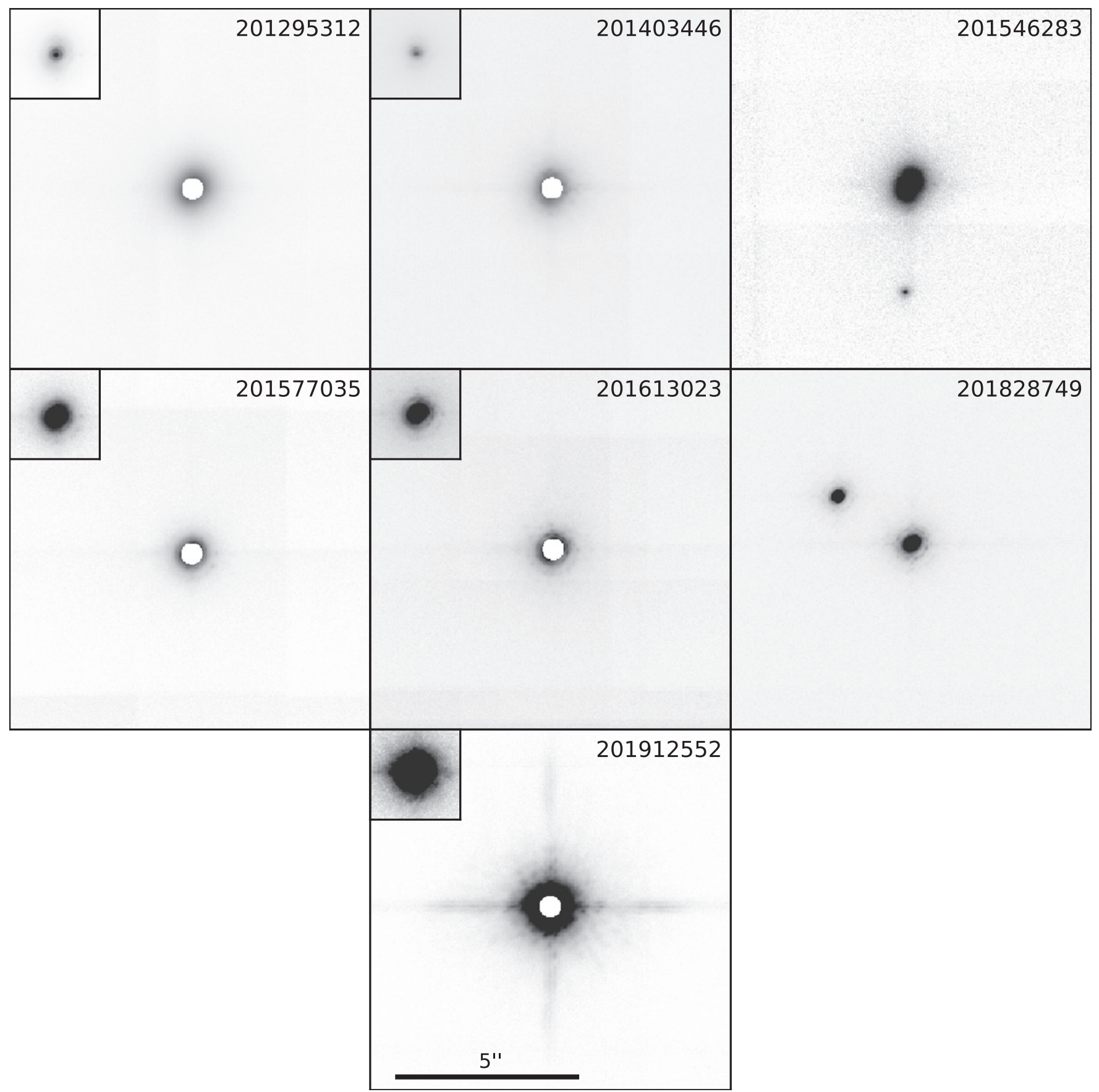

Figure 3. Adaptive optics images for the seven stars observed with high-contrast imaging. The main frame for each single system shows the deep, saturated image. The inset for each single system shows a shallower, unsaturated image to better identify companions at close projected orbital separations. For the two systems with imaged companions, EPIC 201546283 and EPIC 201828749, only unsaturated frames are collected. The pixel scale is 0 " 0252 per pixel. Each subplot is a square 400 pixels on a side and each inset is a square 100 pixels on a side. All subplots, including insets, are plotted on the same scale.

\subsection{Known Background Stars}

The PHARO AO system has a field of view of 25 arcsec. Each $K 2$ pixel is a square, 3." 98 on a side. A background EB within a few $K 2$ pixels of our target stars could mimic a transit signal inside our aperture while evading detection by PHARO. Such wide EBs should appear in seeing-limited ground-based surveys.

To investigate the possibility that such wide companions exist, we query the ninth data release of the Sloan Digital Sky Survey (SDSS DR9, Ahn et al. 2012). For each target, from the depth of the observed transit we determine how bright a background object must be to cause the event if the background object were an equal mass totally eclipsing binary. We then search for all stars within $25^{\prime \prime}$ that are within this brightness limit relative to the candidate host star. All apertures we use in our $K 2$ analysis are smaller than $20^{\prime \prime}$ so this search should encompass the region where possible background contaminants could reside. Of the 31 stars in our sample, eleven have such a companion, plus one detected in AO imaging.

Unlike the original Kepler field, the field for K2 Campaign 1 is well out of the Galactic plane, so the rate of giant, distant background stars is significantly lower. We include all potential 


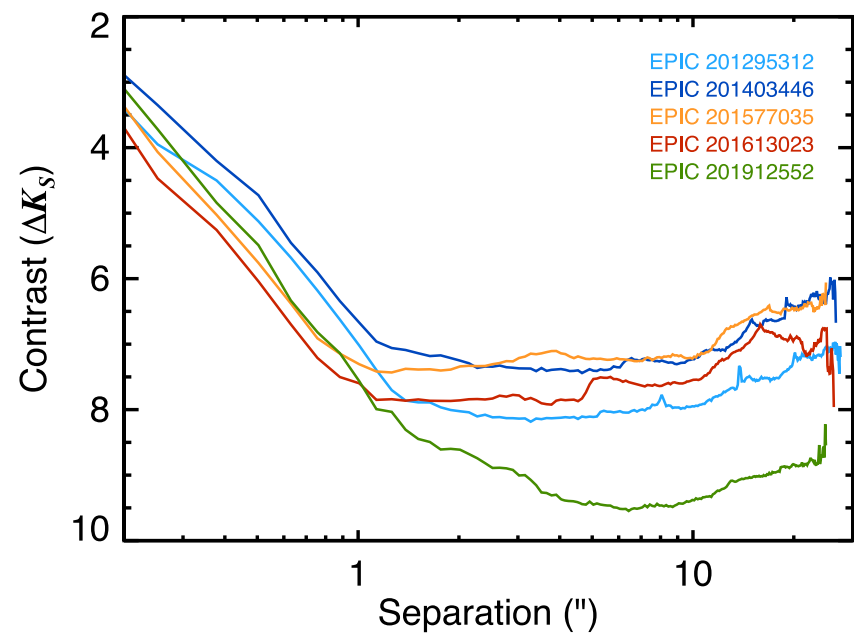

Figure 4. $5 \sigma$ contrast curves for all systems with $\mathrm{AO}$ nondetections. For all systems, we can exclude the possibility that a companion at a given $\Delta K_{S}$ exists. From our known transit depths, we can then rule out significant parameter space in which an eclipsing binary could reside and mimic a transit signal.

contaminants in Table 4. We validate or eliminate each of these as a possibility based on the transit shape. For example, the events near EPIC 201546283 could only be caused by a background binary if the background object was a completely eclipsing system (so that the eclipse depth was 50\%). In this case, the transit would be perfectly V-shaped. Since it is not, this background object likely does not cause the transit event.

In Table 4, the "maximum depth" column represents the maximum observed "transit" depth if the transit were actually caused by a total eclipse of the hypothetical background binary system, inducing a 50\% flux decrement in the background star's apparent brightness.

The photometric apertures used to detect these candidates range in radius from 10 ." 0 to 19 ! 9 . In order to be a plausible contaminant, any companion star must be either within this aperture or just outside but bright enough for signficant flux to leak in. Evaluating each of the systems listed in Table 4, we judge that we cannot yet rule out contamination as a potential source of the transit signal for four candidates: 201295312.01, 201403446.01, 201546283.01, and 201828749.01. Despite receiving low FPP scores from vespa, we list these systems as candidates in Table 5, rather than planets. Further updates to the vespa code will allow consideration of "specific" false positive scenarios; that is, scenarios that correspond to actually detected stars such as these, rather than hypothetical background or bound companions.

The candidates with identified companions that we judge to not be plausible sources of potential contamination are the following.

1. 201546283.01 - for this system, the "maximum depth" is nearly identical to the observed transit depth, which would require a perfectly $\mathrm{V}$-shaped eclipse to explain. As this is not the case (see Figure 2), this signal cannot be caused by contamination from this star.

2. K2-13b (201629650.01) - the companion to this star is 17 ". 3 from the EPIC target. As this is outside the aperture (radius 15."9) and the background star is not particularly bright, we rule out contamination for this system.
3. 201702477.01 - the companion to this star is 12 "' 15 from the EPIC target, and the aperture size is 10 "' 0 . In addition, the maximum depth in this system is almost identical to the transit depth. For these two reasons we rule out contamination in this case.

SDSS is $95 \%$ complete at $r=22.2 \mathrm{mag}$ and the telescope has a PSF of 1." 4 . For the purposes of the vespa calculation, we thus treat nondetection in SDSS data as providing a contrast curve at wide separations down to a limiting magnitude of $r=22.2 \mathrm{mag}$.

\subsection{Archival Imaging}

For the stars with AO nondetections, there is still the possibility that a background binary could be positioned directly behind the target star, evading detection. The probability is small, given the 0 ." 1 diffraction limit of the Hale Telescope at $2 \mu \mathrm{m}$, but nonzero. While the vespa calculations quantify this probability for this to occur, we can also rule out the possibility of such chance alignments, down to a certain contrast, with archival imaging data.

Five of the stars in our sample have proper motions larger

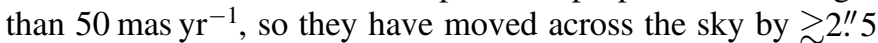
since they were imaged during the first Palomar Observatory Sky Survey (POSS) in the 1950s. To rule out background companions, we download data from the POSS I and II surveys, which imaged these targets in 1952-1955 and 1989-1998, respectively. We also download data from the Sloan Digital Sky Survey, which imaged these fields between 2000 and 2009. As shown in Figure 5, we do not detect any background targets at the present-day location of any of these stars in any of these images.

For this target, we can extend our contrast curves to zero present-day orbital separation and rule out the possibility that these transit events are caused by a background EB. By combining present-day seeing-limited photometric survey data, adaptive optics imaging, and archival photometry, the only stellar companions we would not detect would be those that are gravitationally bound to the target star and positioned in their orbits so that their projected separation is smaller than the diffraction limit of the Hale Telescope. Such an alignment would require the orbital inclination of the binary to be nearly $90^{\circ}$ and the phase $\varpi+\theta \approx \pi / 2$ or $3 \pi / 2$. While we cannot fully rule out this possibility, the vespa calculations confirm that its probability is negligibly small.

\subsection{Tillinghast Reflector Echelle Spectrograph (TRES) Radial Velocities}

We observed EPIC 201912552 on 2015 February 04 and 25 UT with TRES on the $1.5 \mathrm{~m}$ Tillinghast Reflector at the Fred L. Whipple Observatory. These dates were chosen to be near the times of largest RV variations, corresponding to phases of 0.72 and 0.32 relative to the time of transit. The spectra were taken with a resolving power of $R=44,000$ and integration times ranging from 2800 to $3600 \mathrm{~s}$, resulting in $\mathrm{S} / \mathrm{N}$ between 17 and 29 per resolution element.

The spectra were extracted as described in Buchhave et al. (2010). The relative RVs were derived by cross-correlating the spectra against the strongest observed spectrum (in this case, the first) over the wavelength range $4700-6800 \AA$. We selected 19 echelle orders in the analysis, being careful to reject orders 

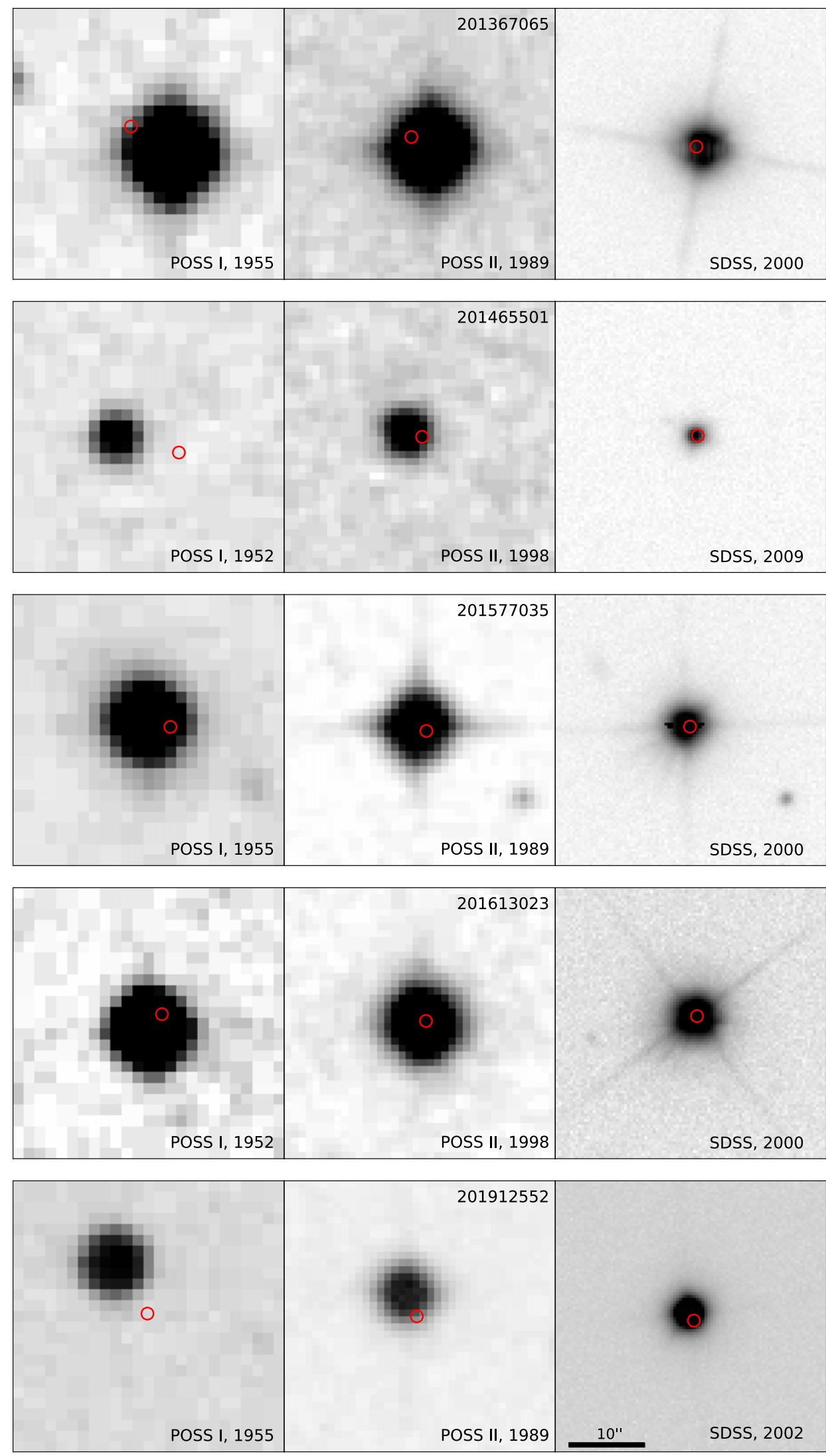

Figure 5. Archival maging for the five highest proper motion targets in our sample. In all cases, there are no background objects directly behind the present day location of the target (red circle) that could be missed by the AO observations. Modern SDSS imaging can also rule out wide companions that may have been missed at wide separations, beyond the AO field of view, such as the companion which can be seen in the images of K2-10 (EPIC 201577035). All figures are aligned such that north is up and east to the left. All subplots are on the same scale. 
with telluric absorption lines, fringing in the far red and those with very low SNR in the blue.

The two observed spectra have RVs that differ by $47 \pm$ $42 \mathrm{~m} \mathrm{~s}^{-1}$. If the RVs were caused by a stellar companion, the RV shift between these observations would be on the order of $\mathrm{km} \mathrm{s}^{-1}$. Therefore, we can rule out any stellar-mass companions that would be able to create this transit signal.

\section{POTENTIALLY INTERESTING SYSTEMS}

\subsection{A Mini-Neptune with Earthlike Insolation}

The planet orbiting K2-18 may be an interesting target for atmospheric studies of transiting exoplanets.

By combining archival and modern seeing-limited data with adaptive optics imaging, we can exclude the possibility these transit events are caused by a background EB. The apparent transits must be caused by an object co-moving with K2-18; radial velocities eliminate the possibility the companion is nonplanetary. Therefore, we confirm the planetary nature of this system.

This star is an M2.8 dwarf at a distance of $34 \pm 4$ pc. Of our planet candidate hosts, only $\mathrm{K} 2-3$ (originally discovered by Crossfield et al. 2015) is brighter in $K$-band. This star is only 0.1 mag fainter in $K$ than GJ 1214 (Charbonneau et al. 2009). Due to the relative brightness of the host star, this target is likely to become a prime target for atmospheric characterization studies and is ideal as a target for future space-based missions such as JWST.

The planet is slightly smaller than GJ $1214 \mathrm{~b}$, but unlike that planet, K2-18b is not highly irradiated. Instead, it is at a reduced semimajor axis $a / R_{\star}=83.8 \pm 9.0$. Its equilibrium temperature is then, assuming zero albedo, $T_{\mathrm{eq}}=272 \pm 15 \mathrm{~K}$, meaning its bulk insolation is $94 \% \pm 21 \%$ that of the Earth's. Although the planet is likely too large to be rocky (Rogers 2015), its atmosphere is likely to be the focus of many future observations, providing a cool analogue to the highly irradiated planets of a similar size found by Kepler.

\subsection{Other Sources of False Positives}

The method of Foreman-Mackey et al. (2015) assumes that all variability in the light curves are caused by either the motion of $K 2$, in which case the variability is shared by all stars, or transits of planets, in which case the variability is intrinsic to only one star. This assumption breaks down for extremely spotted stars where the astrophysical variability is larger than the instrumental magnitude. In that regime, the starspot modulations can be incorrectly fit by the systematic model, causing spurious transits to appear. This appears to be the case with EPIC 201929294, which has coherent starspots that appear to have the same rotation period as the transit period reported previously. Because the starspots are so periodic and coherent, these spurious transits were falsely identified as a planet candidate; we consider that system a false positive in this work.

The candidate object possibly orbiting EPIC 201555883 has a period, time of transit, and transit duration consistent with EPIC 201569483. Such effects are not uncommon in Kepler data. Coughlin et al. (2014) identify 685 KOIs as false positives and outline four physical reasons why these anomalies may occur. EPIC 201555883 is a unique case in that it does not appear to fall under any of these cases. It falls on module 23, while EPIC 201569483 is on module 8, neither $180^{\circ}$ away from nor on the same column as this candidate. Moreover, there is not any evidence of a mechanism that could cause a third star to induce both the appearance of a $7 \%$ eclipse on one module and an additional anomalous transit event on a different module. Instead, this candidate could be a false positive caused by a different systematic mechanism.

Foreman-Mackey et al. (2015) modeled the systematic effects in the $K 2$ light curves using a linear combination of "eigen light curves" generated empirically by running a PCA on the light curves of every star. This means that the training set includes the light curves for variable stars, EBs, and even transiting planets. Again, this star has significant variability caused by starspots. In this case, the fitting procedure tries to account for stellar variability using the eigen light curves. This overfit gives undue weight to eigen light curves that include the transits of EPIC 201569483, causing this spurious transit to occur. Again, we consider this system to be a false positive. As stated in Section 3, by including an empirical Gaussian prior on the weights for the eigen light curves in the linear systematics model, the signals observed on 201555883 and 201929294 are mitigated, suggesting such a scheme should be employed in searching for planet candidates in future campaigns.

The problem of over-fitting stellar variability using eigen light curves can also be solved by adding a stellar activity model to our fitting procedure. In this case, the spacecraft motion could be fit simultaneously with a model of starspot modulation, astroseismic oscillations, and planet transits. Such a model is currently under development (R. Angus et al. 2015, in preparation).

\subsection{Multiple Planet Systems}

Five of the systems reported by Foreman-Mackey et al. (2015) have more than one transiting candidate. One of these is K2-3, a three-planet system originally announced by Crossfield et al. (2015). Another of these is K2-19 (Armstrong et al. 2015), a two-planet system with the orbital periods of the two planets near a $3: 2$ period commensurability. The remaining three are all representative of the multiple-planet systems observed by Kepler (Lissauer et al. 2011; Fabrycky et al. 2014). Two of the systems are near a period commensurability and all three consist of mini-Neptune sized planets.

We do not detect any significant transit timing variations (TTVs) in any of these systems from the $K 2$ data alone. K2-5 (EPIC 201338508) would be expected to have a TTV period of 117 days, but is likely too far from commensurability to have an observable TTV signal. K2-8 (EPIC 201445392) is expected to have a TTV period of 234 days, so this system may be a candidate for additional follow-up to constrain the system masses dynamically. The transiting planets orbiting K216 (EPIC 201754305) are near a 5:2 period commensurability. There is no evidence from Kepler of an abundance of planets near this period ratio, and so this may be coincidence. Followup observations may be warranted to search for an additional planet in this system forming a resonant chain, similar to those observed around other stars (e.g., Swift et al. 2013; Campante et al. 2015).

\subsection{Systems Orbiting Bright Stars}

One of the primary goals of $K 2$ is the detection of transiting planets around bright stars that can be followed up from the 
ground or with future space-based observatories such as JWST (Howell et al. 2014). Of our sample, two systems orbit stars with $K<9$ mag: K2-3 (Crossfield et al. 2015) and K2-18. An additional planet candidate may orbit EPIC 201828749, a star with $K=9.93 \pm 0.03 \mathrm{mag}$. These targets are ideal for groundbased followup and may be useful targets for Spitzer and JWST to probe planetary atmospheres.

\section{RESULTS AND DISCUSSION}

We have presented stellar parameters for all planet candidates systems identified by Foreman-Mackey et al. (2015). We statistically validate 21 of the 36 candidates as bona fide planets, and we identify 6 as false positives, including two systematic false alarms. Of the planets, 4 have been previously validated in other works, while 17 are validated here for the first time. The systems not validated as planets or false positives remain as planet candidates.

Enabling much of this analysis are two new open-source Python packages: isochrones, ${ }^{14}$ which we use to infer posteriors on physical stellar properties based on fitting theoretical stellar models to observed data; and vespa, ${ }^{15}$ a new implementation of the Morton (2012) transit false positive analysis scheme. Both of these packages will continue to be useful in future analysis of transit candidates where comprehensive follow-up observations may be unavailable.

The isochrones package uses the nested sampling scheme MULTINEST to capture the true multimodal nature of the posteriors. Using an MCMC algorithm instead can cause only one peak in the posterior distribution to be sampled. If the photometry is consistent with both a star on the main sequence and the subgiant branch, an MCMC technique could cause one of these peaks (likely the subgiant possibility) to be missed, leading to an underestimation in the likelihood of subgiant stars and and underestimation of the uncertainties of both the stellar and planetary parameters.

With the exception of one object, all of the stellar parameters are derived from comparing photometric observations to the Dartmouth stellar evolution models. As a result, both the stellar and planet parameters are subject to systematic biases induced by discrepancies between the models and reality.

The planets we confirm in this paper, like the planets found in the original Kepler mission, span a wide range of parameter space. They are at distances ranging from 34 to $700 \mathrm{pc}$, have radii ranging from 1.3 to $5.3 R_{\oplus}$, and orbit with periods ranging from 5.0 to 50.3 days. Like the original mission, we find significantly more small planets than large planets, as expected from the radius distributions measured from Kepler (Howard et al. 2012; Fressin et al. 2013; Morton \& Swift 2014).

Unlike the original mission, however, we find that nearly all of our confirmed planets are around stars less massive than the Sun. This difference is a result of both the Campaign 1 field and the target selection process. Campaign 1 is at a significantly higher Galactic latitude than the original Kepler mission, meaning there is a much lower number density of targets at large distances. As massive stars at kiloparsec distances are relatively less likely to exist in Campaign 1 than near the Galactic plane, the pool of targets that could be selected for Campaign 1 contains a larger fraction of subsolar stars.

\footnotetext{
14 http://github.com/timothydmorton/isochrones

15 http://github.com/timothydmorton/vespa
}

Low-mass stars, particularly $M$ dwarfs, are also a specific focus of the $K 2$ mission. One of the primary goals of the Kepler mission was to "determine the abundance of terrestrial and larger planets in or near the habitable zone of a wide variety of spectral types of stars" (Batalha et al. 2013). However, $\sim 70 \%$ of Kepler's target stars had masses within $20 \%$ of the Sun's, while $70 \%$ of the stars in the Galaxy have less than $50 \%$ the mass of the Sun (Brown et al. 2011). K2 will fulfill the promise of Kepler, with the goal of providing a yield of small planets around bright, small stars to facilitate followup measurements (Howell et al. 2014). This is clear from the $K 2$ target selection process, with thousands of $\mathrm{K}$ and $\mathrm{M}$ dwarfs being selected in each campaign. Based on these plans, we expect that $K 2$ will detect hundreds of planets during its lifetime, with the majority being mini-Neptunes and superEarths around stars less massive than the Sun.

We thank Roberto Sanchis-Ojeda (Berkeley), Dan Huber (Sydney) and Jeff Coughlin (SETI) for conversations and suggestions which improved the quality of this manuscript. We also thank Keivan Stassun (Vanderbilt) for his insights into stellar parameters and the rate of subgiant contamination for both Kepler and $K 2$, which significantly improved this work. We thank the anonymous referee for their comments and suggestions. We are grateful to the entire Kepler team, past and present. Their tireless efforts were all essential to the tremendous success of the mission and the successes of $K 2$ present and future. Some of the data presented in this paper were obtained from the Mikulski Archive for Space Telescopes (MAST). STScI is operated by the Association of Universities for Research in Astronomy, Inc., under NASA contract NAS526555. for MAST for non-HST data is provided by the NASA Office of Space Science via grant NNX13AC07G and by other grants and contracts. This paper includes data collected by the Kepler mission. Funding for the Kepler mission is provided by the NASA Science Mission directorate. This paper includes data collected by the Sloan Digital Sky Survey. Funding for SDSS-III has been provided by the Alfred P. Sloan Foundation, the Participating Institutions, the National Science Foundation, and the U.S. Department of Energy Office of Science. The SDSS-III web site is http://www.sdss3.org/. SDSS-III is managed by the Astrophysical Research Consortium for the Participating Institutions of the SDSS-III Collaboration including the University of Arizona, the Brazilian Participation Group, Brookhaven National Laboratory, Carnegie Mellon University, University of Florida, the French Participation Group, the German Participation Group, Harvard University, the Instituto de Astrofisica de Canarias, the Michigan State/ Notre Dame/JINA Participation Group, Johns Hopkins University, Lawrence Berkeley National Laboratory, Max Planck Institute for Astrophysics, Max Planck Institute for Extraterrestrial Physics, New Mexico State University, New York University, Ohio State University, Pennsylvania State University, University of Portsmouth, Princeton University, the Spanish Participation Group, University of Tokyo, University of Utah, Vanderbilt University, University of Virginia, University of Washington, and Yale University. B.T.M. is supported by the National Science Foundation Graduate Research Fellowship under Grant No. DGE-1144469. J.A.J. is supported by generous grants from the David and Lucile Packard Foundation and the Alfred P. Sloan Foundation. D.F. M. and D.W.H. were partially supported by the National 
Science Foundation (grant IIS-1124794), the National Aeronautics and Space Administration (grant NNX12AI50G), and the Moore-Sloan Data Science Environment at NYU. T.D.M. is supported by the National Aeronautics and Space Administration (grant NNX14AE11G).

Facilities: Kepler, Hale(PHARO), FLWO:1.5 m, $\operatorname{IRTF}(\operatorname{SpeX})$

\section{REFERENCES}

Ahn, C. P., Alexandroff, R., Allende Prieto, C., et al. 2012, ApJS, 203, 21 Aldering, G., Adam, G., Antilogus, P., et al. 2002, Proc. SPIE, 4836, 61 Aldering, G., Antilogus, P., Bailey, S., et al. 2006, ApJ, 650, 510 Allard, F., Homeier, D., Freytag, B., et al. 2013, MSAIS, 24, 128 Armstrong, D. J., Veras, D., Barros, S. C. C., et al. 2015, arXiv:1503.00692 Bacon, R., Copin, Y., Monnet, G., et al. 2001, MNRAS, 326, 23 Barentsen, G. 2015, ascl soft, ascl:1503.001

Bastien, F. A., Stassun, K. G., \& Pepper, J. 2014, ApJL, 788, L9 Batalha, N. M., Rowe, J. F., Bryson, S. T., et al. 2013, ApJS, 204, 24 Boisse, I., Bouchy, F., Hébrard, G., et al. 2011, A\&A, 528, A4 Borucki, W. J., Koch, D., Basri, G., et al. 2010, Sci, 327, 977 Borucki, W. J., Koch, D. G., Basri, G., et al. 2011a, ApJ, 728, 117 Borucki, W. J., Koch, D. G., Basri, G., et al. 2011b, ApJ, 736, 19 Bowler, B. P., Liu, M. C., Shkolnik, E. L., \& Tamura, M. 2015, ApJS, 216, 7 Boyajian, T. S., von Braun, K., van Belle, G., et al. 2012, ApJ, 757, 112 Brown, T. M., Latham, D. W., Everett, M. E., \& Esquerdo, G. A. 2011, AJ, 142,112

Buchhave, L. A., Bakos, G. Á, Hartman, J. D., et al. 2010, ApJ, 720, 1118

Burke, C. J., Bryson, S. T., Mullally, F., et al. 2014, ApJS, 210, 19

Campante, T. L., Barclay, T., Swift, J. J., et al. 2015, ApJ, 799, 170

Charbonneau, D., Berta, Z. K., Irwin, J., et al. 2009, Natur, 462, 891

Coughlin, J. L., Thompson, S. E., Bryson, S. T., et al. 2014, AJ, 147, 119

Crossfield, I. J. M., Petigura, E., Schlieder, J. E., et al. 2015, ApJ, 804, 10

Cushing, M. C., Vacca, W. D., \& Rayner, J. T. 2004, PASP, 116, 362

Cutri, R. M., Skrutskie, M. F., van Dyk, S., et al. 2003, 2MASS All Sky Catalog of Point Sources (Pasadena, CA: NASA/IPAC)

Cutri, R. M., Wright, E. L., Conrow, T., et al. 2013, yCat, 2328, 0

Davenport, J. R. A., Ivezić, Z., Becker, A. C., et al. 2014, MNRAS, 440, 3430

Dekany, R., Roberts, J., Burruss, R., et al. 2013, ApJ, 776, 130

Désert, J.-M., Charbonneau, D., Torres, G., et al. 2015, ApJ, 804, 59

Díaz, R. F., Almenara, J. M., Santerne, A., et al. 2014, MNRAS, 441, 983

Dotter, A., Chaboyer, B., Jevremović, D., et al. 2008, ApJS, 178, 89

Fabrycky, D. C., Lissauer, J. J., Ragozzine, D., et al. 2014, ApJ, 790, 146

Feroz, F., Hobson, M. P., \& Bridges, M. 2009, MNRAS, 398, 1601

Ferreira, P. G., \& Jaffe, A. H. 2000, MNRAS, 312, 89

Fitzpatrick, E. L. 1999, PASP, 111, 63
Foreman-Mackey, D., Hogg, D. W., Lang, D., \& Goodman, J. 2013, PASP, 125,306

Foreman-Mackey, D., Montet, B. T., Hogg, D. W., et al. 2015, ApJ, 806, 215 Fressin, F., Torres, G., Charbonneau, D., et al. 2013, ApJ, 766, 81

Gaidos, E., Mann, A. W., Lépine, S., et al. 2014, MNRAS, 443, 2561

Goodman, J., \& Weare, J. 2010, Communications in Applied Mathematics and Computational Science, 5, 65

Grunblatt, S. K., Howard, A. W., \& Haywood, R. D. 2015, arXiv:1501.00369

Hayden, M. R., Bovy, J., Holtzman, J. A., et al. 2015, arXiv:1503.02110

Hayward, T. L., Brandl, B., Pirger, B., et al. 2001, PASP, 113, 105

Haywood, R. D., Collier Cameron, A., Queloz, D., et al. 2014, MNRAS, 443, 2517

Henden, A., \& Munari, U. 2014, CoSka, 43, 518

Howard, A. W., Marcy, G. W., Bryson, S. T., et al. 2012, ApJS, 201, 15

Howell, S. B., Sobeck, C., Haas, M., et al. 2014, PASP, 126, 398

Huber, D., Silva Aguirre, V., Matthews, J. M., et al. 2014, ApJS, 211, 2

Kipping, D. M. 2010, MNRAS, 408, 1758

Lantz, B., Aldering, G., Antilogus, P., et al. 2004, Proc. SPIE, 5249, 146

Lissauer, J. J., Ragozzine, D., Fabrycky, D. C., et al. 2011, ApJS, 197, 8

Mandel, K., \& Agol, E. 2002, ApJL, 580, L171

Mann, A. W., Brewer, J. M., Gaidos, E., Lépine, S., \& Hilton, E. J. 2013a, AJ, 145,52

Mann, A. W., Feiden, G. A., Gaidos, E., Boyajian, T., \& von Braun, K. 2015, ApJ, 804, 64

Mann, A. W., Gaidos, E., \& Ansdell, M. 2013b, ApJ, 779, 188

McCaughrean, M. J., \& Stauffer, J. R. 1994, AJ, 108, 1382

Montet, B. T., Johnson, J. A., Muirhead, P. S., et al. 2015, ApJ, 800, 134

Morton, T. D. 2012, ApJ, 761, 6

Morton, T. D. 2015a, ascl soft, ascl:1503.010

Morton, T. D. 2015b, ascl soft, ascl:1503.011

Morton, T. D., \& Johnson, J. A. 2011, ApJ, 738, 170

Morton, T. D., \& Swift, J. 2014, ApJ, 791, 10

Mullally, F., Coughlin, J. L., Thompson, S. E., et al. 2015, ApJS, 217, 31

Newton, E. R., Charbonneau, D., Irwin, J., \& Mann, A. W. 2015, ApJ, 800,85

Ochsenbein, F., Bauer, P., \& Marcout, J. 2000, A\&AS, 143, 23

Rayner, J. T., Toomey, D. W., Onaka, P. M., et al. 2003, PASP, 115, 362

Rogers, L. A. 2015, ApJ, 801, 41

Rowe, J. F., Coughlin, J. L., Antoci, V., et al. 2015, ApJS, 217, 16

Schlafly, E. F., \& Finkbeiner, D. P. 2011, ApJ, 737, 103

Schlegel, D. J., Finkbeiner, D. P., \& Davis, M. 1998, ApJ, 500, 525

Swift, J. J., Johnson, J. A., Morton, T. D., et al. 2013, ApJ, 764, 105

Thompson, B., Frinchaboy, P., Kinemuchi, K., Sarajedini, A., \& Cohen, R. 2014, AJ, 148, 85

Torres, G., Fressin, F., Batalha, N. M., et al. 2011, ApJ, 727, 24

Vacca, W. D., Cushing, M. C., \& Rayner, J. T. 2003, PASP, 115, 389

Vanderburg, A., Montet, B. T., Johnson, J. A., et al. 2015, ApJ, 800, 59

Zacharias, N., Finch, C. T., Girard, T. M., et al. 2012, yCat, 1322, 0 